\title{
THE REGULAR MODULE PROBLEM. I
}

\author{
T. R. BERGER, B. B. HARGRAVES, AND C. SHELTON
}

Dedicated to Professor Hiroyuki Tachikawa on his sixtieth birthday

\begin{abstract}
In the study of induced representations the following problem arises: Let $H=A G$ be a finite solvable group and $\mathbf{k}$ a field with char $\mathbf{k} \nmid|A|$. Let $V$ be an irreducible, faithful, primitive $\mathbf{k}[A G]$-module. Suppose $H$ contains a normal extraspecial $r$-subgroup $R$ with $Z(R) \leq Z(H)$ and that $A$ acts faithfully on $R$. Under what conditions does $A$ have a regular direct summand in $V$ ?

In this paper we consider this question under the hypotheses that $G=M R$, where $1 \neq M$ is normal abelian in $A M, A$ is nilpotent, $(|A|,|M R|)=$ $(|M|,|R|)=1$, and $R / Z(R)$ is a faithful, irreducible $A M$-module. We show that $A$ has at least three regular direct summands in $V$ unless $|A|, \exp (M)$, and $r$ satisfy certain very restrictive conditions.
\end{abstract}

Let $H=A G$ be a finite solvable group, where $A$ is nilpotent, $G$ is normal, and $(|A|,|G|)=1$. Let $k$ be a field of characteristic not dividing $|A|$ and $V$ a $k[H]$-module. Assume that $V$ is faithful and irreducible. The study of induced representations often leads to the following two questions.

(1) (Regular Orbit Problem) Under what conditions does $A$ have a regular orbit on $V$ ?

(2) (Regular Module Problem) Suppose that $H$ contains a normal extraspecial $r$-group $R \leq G$, where $Z(R) \leq Z(H)$ and $A$ acts faithfully on $R$, and that $V$ is primitive. Under what conditions does $A$ have a regular direct summand in $V$ ?

A discussion of the applications of answers to these questions is contained in [8].

The Hall-Higman series of papers [1-7] considers these questions when $A$ is wreath free. However, general results on minimal cases are given for the regular orbit problem in [1] and for the regular module problem in [2,3]. General methods for reduction to minimal cases are studied in [5]. These results are used to study the regular orbit problem in [14] where a general solution is given for a nilpotent group $A$. Here we wish to begin the extension of these results to the regular module problem.

The regular module problem is always more difficult than the regular orbit problem because there are many more groups for which there is no regular

Received by the editors June 12, 1989 and, in revised form, May 25, 1990.

1980 Mathematics Subject Classification (1985 Revision). Primary 20C15.

The first author's research was partially supported by a National Science Foundation grant.

The second author's research was partially supported by a faculty development grant from Gustavus Adolphus College. 
direct summand and because these exceptional groups are far more complex. The Hall-Higman series [1-7] describes all the essential minimal exceptional groups in the case where $G$ acts cyclically on $R / Z(R)$. This paper extends these results to the case where the action of $G$ on $R / Z(R)$ is abelian.

The inductive reductions used in this paper allow one to reduce to minimal cases where the action of $H$ on $R / Z(R)$ is form primitive. Therefore, in this paper we are starting with the cases where $G$ acts essentially as a cyclic group on $R / Z(R)$. In future papers we will consider the cases starting where $G$ acts essentially as an extraspecial group. These results are required for a general answer to the regular module problem.

The exceptional groups (i.e., those with no regular direct summand in $V$ ) all arise when certain interactions occur between the primes dividing $|A|$ and those dividing $|G|$. These may be explicitly excluded as is done in Shult [19] or implicitly excluded as in Turull [20]. We are attempting here to give methods for examining the exceptional cases excluded by these types of hypotheses. Our justification is that most of the interesting pathological behavior in representation theory occurs for the exceptional groups we are studying.

Because our rationale is to examine the pathology and because this pathology is plentiful, the computations are often elaborate and finicky. Wherever it seemed reasonable, we have suppressed the computations, indicating how they were done.

The main result is obtained through a sequence of reductions. We have isolated each of these reductions via a sequence of propositions. Thus, the hypotheses of each proposition are set to handle one particular reduction. The main theorem of this paper is:

Theorem. Suppose $H=A M R$, where $R$ is a normal extraspecial $r$-subgroup for a prime $r, 1 \neq M$ is a normal abelian subgroup of $A M, A$ is nilpotent, and $(|A|,|M R|)=(|M|,|R|)=1$. Assume that $Z(R) \leq Z(H)$ and that $\bar{R}=$ $R / Z(R)$ is a faithful irreducible AM-module. Let $\lambda \neq 1$ be a complex linear character of $Z(R)$ and $V$ be a complex $H$-module lying over $\lambda$. Then $\left.V\right|_{A}$ contains a direct sum of three copies of the regular A-module unless:

(1) $M$ is cyclic, $R$ is a form quasiprimitive module, and $(A, M, R)$ is one of the exceptional groups $(A, N, R)$ given in Theorem (3.13).

(2) $A$ is a 2-group and one of the following occurs:

(i) $\exp (M)=3$ and $r=3 \cdot 2^{a} \pm 1$ for some $a$, or

(ii) $\exp (M)=5$ and either $r=3$ or $r=5 \cdot 2^{a} \pm 1$ for some $a$, or

(iii) $\exp (M)$ divides $(r-1) / 2$ and $r=2^{a}-1$ for some $a$, or

(iv) $\exp (M)=15$ and $r=11$.

(3) $\mathscr{O}_{2^{\prime}}(A)$ is cyclic, both $\left|\mathscr{O}_{2^{\prime}}(A)\right|$ and $\exp (M)$ divide $(r+1) / 2$, and $r=$ $2^{a}+1$ for some $a$.

(4) $A$ is a p-group for an odd prime $p, \exp (M)=2$, and $r^{a}=2 \cdot p^{b}-1$ for some $a$ and $b$.

For each exceptional conclusion there is at least one example $H$ so that $V$ does not contain three regular $A$-modules. This theorem is most useful in an inductive setup. For the inductive setup to work easily, it is necessary to know that $V$ contains at least three regular $A$-direct summands (rather than just one).

We have divided this paper as follows. $\S 1$ is a compendium of notation. 
$\S 2$ sets up the notation for the canonical computations. $\S 3$ collects notation, definitions, and results from previous papers on this subject. We have found it necessary to extend some of these results in minor ways. $\S 4$ is devoted to tensor induction and the related reduction results we will need. $\$ 5$ contains all the reduction steps mentioned earlier. Finally, in $\S 6$ we carefully give our hypotheses, the main theorem, and its proof.

\section{REMARKS AND NOTATION}

All groups considered are finite and solvable. The following standard notation is used.

(1.1) $G^{(n)}=G \times G \times \cdots \times G$ ( $n$ factors).

(1.2) $G^{\Delta} \leq G^{(n)}$ is defined by $G^{\Delta}=\{(g, g, \ldots, g): g \in G\}$.

(1.3) $S_{n}$ is the symmetric group on $n$ letters.

(1.4) Let $H \leq S_{n}$. The wreath product $G \sim H$ is defined as the semidirect product $G^{(n)} H$ with the action of $h \in H$ on $f \in G^{(n)}$ given by $f^{h}(i)=$ $f(h(i)), i=1,2, \ldots, n$.

(1.5) $G^{\prime}$ always refers to the derived group of $G$.

(1.6) $G^{2}=\left\{g \in G: g=x^{2}\right.$ for some $\left.x \in G\right\}$.

(1.7) $1_{G}$ and $\rho_{G}$ refer to the trivial and regular characters of $G$, respectively. Also, if $N \triangleleft G$, then every $G / N$-module (-character) can be viewed as a $G$ module (-character). In particular, $\rho_{G / N}$ is a $G$-character.

(1.8) $V_{1}+V_{2}$ and $V_{1} \oplus V_{2}$ refer to the internal and external direct sums of $V_{1}$ and $V_{2}$, respectively.

(1.9) $\delta_{i j}$ is the Kronecker delta, i.e., $\delta_{i j}=1$ if $i=j$ and $\delta_{i j}=0$ if $i \neq j$.

(1.10) For $g \in G,|g|_{2}$ is the 2-part of the order of $g$. That is, if $|g|=2^{a} b$, $b$ odd, then $|g|_{2}=2^{a}$.

Let $G$ be a group, $k$ a field, $V$ a $k[G]$-module, and $\chi$ the character of $G$ on $V$. Then

$$
V^{(n)}=V \otimes V \otimes \cdots \otimes V \quad(n \text { factors })
$$

is naturally a $\left(G \sim S_{n}\right)$-module, where for $\left(g_{1}, g_{2}, \ldots, g_{n}\right) \in G^{(n)}$ and for $y \in S_{n}$ the action of $y\left(g_{1}, g_{2}, \ldots, g_{n}\right)$ on $\left(v_{1} \otimes v_{2} \otimes \cdots \otimes v_{n}\right) \in V^{(n)}$ is given by

$$
\begin{gathered}
y\left(g_{1}, g_{2}, \ldots, g_{n}\right)\left(v_{1} \otimes v_{2} \otimes \cdots \otimes v_{n}\right)=y\left(g_{1} v_{1} \otimes g_{2} v_{2} \otimes \cdots \otimes g_{n} v_{n}\right) \\
=\left(g_{y^{-1}(1)} v_{y^{-1}(1)}\right) \otimes\left(g_{y^{-1}(2)} v_{y^{-1}(2)}\right) \otimes \cdots \otimes\left(g_{y^{-1}(n)} v_{y^{-1}(n)}\right) .
\end{gathered}
$$

By $\chi^{(n)}$ we mean the $\left(G \sim S_{n}\right)$-character of $V^{(n)}$.

Notice that even if $V$ is a faithful $G$-module, it is not necessarily true that $V^{(n)}$ is a faithful $\left(G \sim S_{n}\right)$-module. For example, if $V$ is faithful and irreducible and $g \in G$ acts like -1 on $V$, then $(g, g)\left(v_{1} \otimes v_{2}\right)=\left(-v_{1}\right) \otimes\left(-v_{2}\right)=$ $\left(v_{1} \otimes v_{2}\right)$ and $(g, g)$ acts trivially on $V^{(2)}$.

Recall that for a group $G$ the values of the regular $G$-character are given by $\rho_{G}(1)=|G|$ and $\rho_{G}(g)=0$ for $g \in G^{\#}$ and that $\rho_{G}=\sum \mu(1) \mu$, where the sum is over the irreducible characters $\mu$ of $G$. Suppose $V$ is a $G$-module with character $\chi$. We will frequently want to show that $\chi \geq n \rho_{G}$, and hence that $V$ contains $n$ copies of the regular $G$-module. One method of doing this is to show that $(\chi, \mu) \geq n \mu(1)$ for all irreducible characters $\mu$ of $G$. Another method is to write $\chi=n \rho_{G}+\psi$, where $\psi$ is a sum of $G$-characters. While it 
may be difficult to find $\psi$, it is easy to determine if it is correct. One merely evaluates $n \rho_{G}+\psi$ for $g \in G$ and compares the result to $\chi(g)$.

\section{THE MOdUle $V_{\lambda}$ AND CHARACTER $\chi_{\lambda}$}

Throughout this section we will have $G=A R$ a finite group with normal extraspecial $r$-subgroup $R$ ( $r$ a prime) and $Z(R) \leq Z(G)$. Then $\bar{R}=R / Z(R)$ is a vector space over the field $\operatorname{GF}(r)$ and $A$ acts on $\bar{R}$ as automorphisms. We begin this section with a discussion of the ordinary characters of extraspecial groups and then consider extensions to groups with normal extraspecial subgroups. In particular, certain canonical character extensions will be defined and methods given for explicitly computing character values.

(2.1) Theorem [15, V.16.14]. If $R$ is extraspecial of order $r^{2 n+1}$, then $R$ has precisely the following irreducible characters:

(1) $r^{2 n}$ linear characters, lifted from $\bar{R}=R / R^{\prime}$ and

(2) $r-1$ faithful characters of degree $r^{n}$, which arise in the following way. Let $M<R$ be a maximal abelian normal subgroup (of order $r^{n+1}$ ) and $\lambda \neq 1$ one of the $r-1$ distinct linear characters of $Z(R)$. Then $\lambda$ has an extension $\mu$ to $M$, with $\chi=\left.\mu\right|^{R}$ irreducible of degree $r^{n}$ and $\left.\chi\right|_{Z(R)}=\chi(1) \lambda$. This determines a one-to-one correspondence between the nontrivial $Z(R)$-characters and the nonlinear irreducible $R$-characters.

If $\chi$ is a $G$-character with associated representation $X$, then $\operatorname{det} \chi$ is the linear character defined by the determinant of $X$. That is, $\operatorname{det} \chi(g)=\operatorname{det} X(g)$, $g \in G$.

(2.2) Theorem [3, 2.2, 2.3]. Suppose $G=A R$ is the semidirect product of the normal extraspecial r-group $R$ by $A$, with $(|A|, r)=1$ and $Z(R) \leq Z(G)$. Assume that $k$ is a field of characteristic 0 containing the $|G|$ th roots of unity. Let $\lambda \neq 1$ be a linear $k$-character of $Z(R)$. Then there exists a unique $k[G]-$ module $V_{\lambda}(A R)$ with associated character $\chi_{\lambda}=\chi_{\lambda}(A R)$ such that

(1) $\left.\chi_{\lambda}\right|_{Z(R)}$ is a multiple of $\lambda$,

(2) $\left.\chi_{\lambda}\right|_{R}$ is irreducible, and

(3) $\left.\operatorname{det} \chi_{\lambda}\right|_{A}$ is trivial.

Further, if $B \leq A$, then $\chi_{\lambda}(B R)=\left.\chi_{\lambda}(A R)\right|_{B R}$. In particular, for $x \in A$, $\chi_{\lambda}(A R)(x)=\chi_{\lambda}(\langle x\rangle R)(x)$.

Note that the last statement indicates that the values of $\chi_{\lambda}$ are determined locally. In $[3,2.4-2.8]$ the method of computing $\chi_{\lambda}(\langle x\rangle R)(x)$ is given. We summarize the results.

(2.3) Theorem. Suppose $G=A R$ is the semidirect product of the extraspecial $r$-subgroup $R$ by the cyclic group $A=\langle x\rangle$. Assume further that $(|A|, r)=1$, $Z(R) \leq Z(G)$, and $C_{A}(R)=1$. Let $k$ be a field of characteristic 0 containing the $|G|$ th roots of unity. If $\lambda \neq 1$ is a linear character of $Z(R)$, then $\chi_{\lambda}(x)$ may be determined as follows:

(1) Write $\left.\bar{R}\right|_{\langle x\rangle}=\sum_{\mathscr{C}}^{\oplus} \bar{R}_{C}$, where, for each subgroup $C$ of $\langle x\rangle, \bar{R}_{C}$ is the sum of all submodules of $\bar{R}$ with kernel $C$, and $\mathscr{C}$ is the set of all subgroups $C \leq\langle x\rangle$ for which $\bar{R}_{C} \neq(0)$. The $\bar{R}_{C}$ 's are mutually orthogonal nonsingular spaces. So each $\bar{R}_{C}$ has even dimension $2 n_{C}$. 
(2) For each $C \in \mathscr{C}$ there is a sign $\varepsilon_{C} \quad(= \pm 1)$ satisfying

$$
r^{n_{C}} \equiv \varepsilon_{C} \bmod [\langle x\rangle: C] .
$$

(3) Put

$$
\gamma_{C}= \begin{cases}(-1)^{\left(r^{n} C-\varepsilon_{c}\right) /[\langle x\rangle: C]} & \text { if }[\langle x\rangle: C] \text { is even } \\ 1 & \text { otherwise. }\end{cases}
$$

Then

$$
\chi_{\lambda}(x)=r^{n} \prod_{\mathscr{C} *} \varepsilon_{C} \gamma_{C},
$$

where $\operatorname{dim}\left(\bar{R}_{\langle x\rangle}\right)=2 n$ and $\mathscr{C}^{*}=\mathscr{C} \backslash\{\langle x\rangle\}$.

When applying this theorem it is necessary to determine the dimensions of the modules $\bar{R}_{C}$. The groups for which we will need to do this will satisfy the hypotheses of the following lemma.

(2.4) Lemma. Suppose $R \triangleleft A R$ is an extraspecial $r$-group, $r$ an odd prime, $A$ is a 2-group, and $Z(R) \leq Z(A R)$. Assume $\bar{R}=R / Z(R)$ is a faithful irreducible $A$-module. Then $Z(A)$ is cyclic. Let $z \in A$ be the unique central involution. Suppose we have $x z$ conjugate to $x$ for all $x \in A$ with $\langle x\rangle \cap Z(A)=1$. Then for all such $x$,

$$
\operatorname{dim} C_{\bar{R}}(x)=(\operatorname{dim} \bar{R}) /|x| .
$$

Proof. The proof is by mathematical induction on $|x|$. We can write $\bar{R}=$ $\bar{R}_{1} \dot{+} \bar{R}_{2}+\bar{R}_{3}$, where $x$ acts like 1 on $\bar{R}_{1}=C_{\bar{R}}(x), x$ acts like -1 on $\bar{R}_{2}$, and $x^{2}$ is faithful on $\bar{R}_{3}$. Now $x z$ acts like -1 on $\bar{R}_{1}$ and like 1 on $\bar{R}_{2}=$ $C_{\bar{R}}(x z)$. Since $x z$ is conjugate to $x, \operatorname{dim} C_{\bar{R}}(x)=\operatorname{dim} C_{\bar{R}}(x z)$. That is, $\operatorname{dim} \bar{R}_{1}=\operatorname{dim} \bar{R}_{2}$. Since by induction

$$
\operatorname{dim}\left(\bar{R}_{1} \dot{+} \bar{R}_{2}\right)=\operatorname{dim} C_{\bar{R}}\left(x^{2}\right)=(\operatorname{dim} \bar{R}) /\left|x^{2}\right|,
$$

we have $\operatorname{dim} \bar{R}_{1}=(\operatorname{dim} \bar{R}) /|x|$ as desired.

\section{FORM QUASIPRIMITIVE MODULES}

In this section we first discuss induction of general form quasiprimitive modules. The theory parallels that of ordinary induction of primitive and quasiprimitive modules. We then look at $V_{\lambda}(A M R)$ when $R / Z(R)$ is a form quasiprimitive $A M$-module. (In $[5, \S 7]$ these modules are called minimal.) We will especially investigate the situations in which $\left.\chi_{\lambda}(A M R)\right|_{A} \nsupseteq \rho_{A}$.

(3.1) Hypothesis. Let $k$ be a finite field, $G$ a group, and $V$ a $k[G]$-module. Suppose $g: V \times V \rightarrow k$ is a nonsingular symplectic form on $V$ fixed by $G$.

We say $V$ is form induced by $U$ if there is a subgroup $H$ of $G$ and a nonsingular $k[H]$-module $U$ such that $\left.V \simeq U\right|^{G}$ and the distinct submodules among the $x U, x \in G$, are pairwise orthogonal. An irreducible module which is not form induced is said to be form primitive. We also need to define the notion of a form quasiprimitive module.

(3.2) Definition. Assume Hypothesis (3.1). We say $V$ is form quasiprimitive (or minimal) if for any normal subgroup $N$ of $G$ either

(1) $\left.V\right|_{N}$ is homogeneous, or 
(2) $\left.V\right|_{N}=W_{1} \oplus W_{2}$, where the $W_{i}$ are the homogeneous components and are totally isotropic. Then $N$ acts contragrediently on $W_{1}$ and $W_{2}$.

(3.3) Proposition [5, 7.8]. Assume Hypothesis (3.1). If $V$ is form primitive, then $V$ is form quasiprimitive.

(3.4) Corollary. Assume Hypothesis (3.1). Then $V$ is induced from a form quasiprimitive module.

(3.5) Hypothesis. Let $R \triangleleft A M R$, where $R$ is an extraspecial $r$-group, $r$ is a prime, $M \triangleleft A M$, and $(|A M|, r)=(|A|,|M|)=1$. Assume $Z(R) \leq Z(A M R)$ and $\bar{R}=R / Z(R)$ is an irreducible $A M$-module.

Then the commutator map on $R$ induces a nonsingular symplectic form and we have

(3.6) Corollary. Assume Hypothesis (3.5). Then there are groups $R_{0} \leq R$, $M_{0} \leq M$, and $A_{0} \leq A$ such that $M_{0} \triangleleft A_{0} M_{0}, Z(R) \leq R_{0} \triangleleft A_{0} M_{0} R_{0}, \bar{R}_{0}=$ $R_{0} / Z(R)$ is a form quasiprimitive $A_{0} M_{0}$-module, and $\bar{R}=R / Z(R)$ is form induced from $\bar{R}_{0}$, i.e., $\left.\bar{R} \simeq \bar{R}_{0}\right|^{A M}$, where $\bar{R}_{0}$ is viewed as an $A_{0} M_{0}$-module and for $x \in A M$ either $x^{-1} R_{0} x=R_{0}$ or $\left[x^{-1} R_{0} x, R_{0}\right]=1$. Also, $R_{0}$ is extraspecial since $\bar{R}_{0}$ is nonsingular.

(3.7) Hypothesis. Assume $A N R$ satisfies Hypothesis (3.5) (with $N=$ " $M$ "), $1 \neq N$ is cyclic, $A$ is nilpotent, and $\bar{R}=R / Z(R)$ is a faithful quasiprimitive $A N$-module. Write $|R|=r^{2 m+1}$.

(3.8) Theorem [2, 3.20; 3, 4.12]. Assume Hypothesis (3.7). Then there is an identification so that $A N \leq B$ and $\bar{R}$ is given by one of the examples (3.9), (3.10), (3.11), or (3.12) below. In these examples $g$ is the symplectic form induced by the commutator on $R$. Further, if $A N$ is nilpotent, then $A N \leq B$ is given by either (3.9) or (3.10).

Let $k=\mathrm{GF}(r), \tilde{k}=\mathrm{GF}\left(r^{m}\right)$, and $\hat{k}=\mathrm{GF}\left(r^{2 m}\right)$, and let $\operatorname{Tr}: \tilde{k} \rightarrow k$ be the trace map.

(3.9) Example. $B$ is cyclic of order $r^{m}+1$.

Let $\bar{R}=\hat{k}^{+}$and $\varphi: \varepsilon \rightarrow \varepsilon^{r^{m}}$, the automorphism of order 2 of $\hat{k}$. Let $B$ be the multiplicative subgroup of $\hat{k}^{\times}$of order $r^{m}+1$. Then $B$ acts on $\bar{R}$ by multiplication. Choose $\mu \in \hat{k}^{\times}$so that $\mu^{\varphi}=-\mu$ (if $r=2$, set $\mu=1$ ) and for $u, v \in \bar{R}$ set $g(u, v)=\operatorname{Tr}\left(\mu\left(u v^{\varphi}-u^{\varphi} v\right)\right)$.

(3.10) Example. $B=S \times C$, where $S$ is semidihedral of order $2^{t+1}, 2^{t} \| r^{m}-1$, and $N \leq C$ which is cyclic of odd order $\left(r^{n}+1\right) / 2, m=2 n$, and $r^{n} \equiv 1 \bmod 4$.

Let $\bar{R}$ be a two-dimensional $\tilde{k}$-space with basis $\left\{e_{1}, e_{2}\right\}$. As a $\tilde{k}$-space we write matrices in the basis $\left\{e_{1}, e_{2}\right\}$ to represent linear (or semilinear) transformations. Choose $\nu \in \tilde{k}^{\times}$of order $2^{t}$ and $\theta \in \tilde{k}^{\times}$of order $\left(r^{n}+1\right) / 2$. Let $\psi: \varepsilon \rightarrow \varepsilon^{r^{n}}$ be the automorphism of order 2 of $\tilde{k}$. Set

$$
S=\left\langle\left[\begin{array}{ll}
\nu & \\
& \nu^{-1}
\end{array}\right],\left[\begin{array}{cc} 
& \psi \\
-\psi &
\end{array}\right]\right\rangle
$$

and 


$$
C=\left\langle\left[\begin{array}{ll}
\theta & \\
& \theta^{-1}
\end{array}\right]\right\rangle \text {. }
$$

For $u=\alpha e_{1}+\beta e_{2}$ and $v=\alpha^{\prime} e_{1}+\beta^{\prime} e_{2}$, where $\alpha, \beta, \alpha^{\prime}, \beta^{\prime} \in \tilde{k}$, set $g(u, v)=$ $\operatorname{Tr}\left(\alpha \beta^{\prime}-\alpha^{\prime} \beta\right)$.

For $k_{0}$ an extension field of $k$, let $\mathscr{G}=\mathscr{G}\left(k_{0} / k\right)$ be the Galois group of $k_{0}$ over $k$. Form the semidirect product $\mathscr{T}\left(k_{0} / k\right)=\mathscr{G} k_{0}^{\times}$. The additive group $k_{0}^{+}$is naturally a $\mathscr{T}\left(k_{0} / k\right)$-module with action $(\sigma x)(v)=(x v)^{\sigma^{-1}}$ for $\sigma \in \mathscr{G}$, $x \in k_{0}^{\times}$, and $v \in k_{0}^{+}$. We use the group $\mathscr{T}\left(k_{0} / k\right)$ in Examples (3.11) and (3.12).

(3.11) Example. $B$ equals the group $\mathscr{T}_{1}$ given below.

Let $\bar{R}=\hat{k}^{+}, \varphi, \mu$, and $g$ be as in Example (3.9). Let $\nu \in \hat{k}^{\times}$have order $r^{m}+1,\langle\sigma\rangle=\mathscr{G}(\hat{k} / k)$, and choose $\alpha \in \hat{k}^{\times}$such that $\mu^{\sigma} \mu^{-1} \alpha^{\varphi} \alpha=1$. Set $\mathscr{T}_{1}=\langle\sigma \alpha, \nu\rangle$. ( $\mathscr{T}_{1}$ is the subgroup of $\mathscr{T}(\hat{k} / k)$ that fixes the form $g$.) Here $N \leq\langle\nu\rangle$.

(3.12) Example. $B$ equals the group $\mathscr{T}_{2}$ below.

Let $\bar{R}$ and $g$ be as in Example (3.10), $\langle\sigma\rangle=\mathscr{G}(\tilde{k} / k)$, and $\langle\nu\rangle=k^{\times}$. (No assumptions are made about $m$ or congruence mod 4 .) Set

$$
\mathscr{T}_{2}=\left\langle\left[\begin{array}{ll} 
& 1 \\
-1 &
\end{array}\right],\left[\begin{array}{ll}
\sigma & \\
& \sigma
\end{array}\right], \nu^{\prime}=\left[\begin{array}{ll}
\nu & \\
& \nu^{-1}
\end{array}\right]\right\rangle .
$$

Here $N \leq\left\langle\nu^{\prime}\right\rangle$.

(3.13) Theorem [3, 3.8, 4.28]. Assume Hypothesis (3.7). Let $\lambda \neq 1$ be a linear $Z(R)$-character and let $V_{\lambda}(A N R)$ be the module of $(2.2)$. Then $V_{\lambda}(A N R)$ contains three copies of the regular $A$-module unless $A \leq \widehat{A}$, where $\widehat{A}$ (= " $A$ "), $N$, and $R$ also satisfy Hypothesis (3.7) and we have one of the entries of Table (3.14).

Remarks. When $\widehat{A} N$ is nilpotent $[2,3.20]$ applies. When $\widehat{A} N$ is as in Example (3.9), $\left.\chi_{\lambda}\right|_{\widehat{A}}=(|N|-1) \rho_{\widehat{A}}+\left(\rho_{\widehat{A}}-\mu\right)$, where $\mu$ is a linear character. Since $\left.\chi_{\lambda}\right|_{\widehat{A}} \nsucceq 3 \rho_{\widehat{A}}$ we must have $|N|=2$ or 3 and we obtain exceptions (1) and (2). When $\widehat{A} N$ is as in Example (3.10), then $\left.\chi_{\lambda}\right|_{\widehat{A}} \geq|N|\left[\left(r^{n}-1\right) / 2^{t}-1 / 2\right] \rho_{\widehat{A}}$, where $2^{t-1} \| r^{n}-1$. Since $|N| \geq 3$ and $\left.\chi_{\lambda}\right|_{\widehat{A}} \nsucceq 3 \rho_{\widehat{A}}$ we must have $r^{n}-1=2^{t-1}$ and we obtain exceptions (3) and (4). The remaining exceptions are obtained from the table of exceptions of $[3,4.28]$, which applies when $\widehat{A} N$ is not nilpotent. Here the conditions on $|A|,|N|$, and $|R|$ are a bit more stringent and so some of the exceptions to that theorem do not appear here. Also, each of the possibilities for $A \leq \widehat{A}$ is listed separately there, while here only $\widehat{A}$ is given.

The cases in which $\left.V_{\lambda}(\widehat{A} N R)\right|_{\widehat{A}}$ do not contain any regular $\widehat{A}$-modules will lead to most of the difficulties which arise later. We will need to do explicit character computations in these cases. We now describe these groups explicitly and give the values of the characters $\left.\chi_{\lambda}(\widehat{A} N R)\right|_{\widehat{A}}$. 
TABLE (3.14)

\begin{tabular}{|c|c|c|c|c|c|c|c|}
\hline & $r$ & $m$ & $|\widehat{A}|$ & $|N|$ & example & \#reg & comments \\
\hline$(1)$ & $r$ & $m$ & $\left(r^{m}+1\right) / 2$ & 2 & 3.9 & 1 & \\
\hline$(2)$ & $r$ & $m$ & $\left(r^{m}+1\right) / 3$ & 3 & 3.9 & 2 & \\
\hline (3) & 3 & 4 & 32 & 5 & 3.10 & 0 & \\
\hline (4) & $2^{s}+1$ & 2 & $2^{s+2} \cdot f$ & $g$ & 3.10 & 0 & $s \geq 2,{ }^{*},{ }^{* *}$ \\
\hline$(5)$ & 3 & 2 & 8 & 5 & 3.11 & 1 & \\
\hline (6) & $3 \cdot 2^{s}-1$ & 1 & $2^{s+1}$ & 3 & 3.11 & 1 & \\
\hline (7) & 2 & 5 & $3 \cdot 5$ & 11 & 3.11 & 2 & \\
\hline$(8)$ & 5 & 3 & $2 \cdot 27$ & 7 & 3.11 & 2 & \\
\hline (9) & $5 \cdot 2^{s}-1$ & 1 & $2^{s+1}$ & 5 & 3.11 & 2 & \\
\hline (10) & 3 & 4 & 128 & 5 & 3.12 & 0 & \\
\hline (11) & $2^{s}+1$ & 2 & $2^{s+3}$ & $g$ & 3.12 & 0 & $s \geq 2, *$ \\
\hline (12) & $2^{s}-1$ & 2 & $2^{s+3}$ & $g$ & 3.12 & 0 & $s \geq 3,{ }^{*}$ \\
\hline (13) & 5 & 4 & $32 \cdot 13$ & 3 & 3.12 & 1 & \\
\hline (14) & 7 & 4 & $64 \cdot 25$ & 3 & 3.12 & 1 & \\
\hline (15) & $3 \cdot 2^{s}+1$ & 1 & $2^{s+1}$ & 3 & 3.12 & 1 & \\
\hline (16) & 3 & 8 & $64 \cdot 41$ & 5 & 3.12 & 2 & \\
\hline (17) & 11 & 2 & 32 & $3,5,15$ & 3.12 & 2 & \\
\hline (18) & $3 \cdot 2^{s}+1$ & 2 & $2^{s}(r+1)$ & 3 & 3.12 & 2 & \\
\hline (19) & $5 \cdot 2^{s}+1$ & 1 & $2^{s+1}$ & 5 & 3.12 & 2 & \\
\hline (20) & $5 \cdot 2^{s}+1$ & 2 & $2^{s+1}(r+1)$ & 5 & 3.12 & 2 & \\
\hline
\end{tabular}

${ }^{*}: 1 \neq g$ is odd and divides $r^{m}-1$.

${ }^{* *}: 1 \leq f$ is also odd and divides $r+1$. Also, $(f, g)=1$.

\# reg gives the number of regular $\hat{A}$ modules in $V_{\lambda}(\hat{A} N R)$.

$\widehat{A}$ is the group described in the indicated example.

(3.15) Theorem. Assume Hypothesis (3.7). Let $A_{0} \leq A$. Assume $\left.\chi_{\lambda}(A N R)\right|_{A_{0}} \geq$ $\rho_{A_{0}}$. Then $A_{0} \leq A \leq \hat{A}$, where $\hat{A}, N$, and $R$ also satisfy Hypothesis (3.7) and we have one of (1), (2), (3), or (4) below. Further, in each of these cases $\left.\chi_{\lambda}(\widehat{A} N R)\right|_{\widehat{A}} \geq \frac{1}{2}\left(\rho_{\widehat{A}}-\rho_{\widehat{A} / \widehat{A}^{\prime}}\right)$.

(1) $\hat{A}=C \times\left\langle h, k: h^{2^{s}}=k^{2}=-1, h^{k}=-h^{-1}\right\rangle$, where $C$ is cyclic of odd order dividing $\left(r^{m / 2}+1\right) / 2, r^{m / 2}=2^{s}+1$, and $\left\langle h^{2}, h k\right\rangle<A_{0}$. For $x \in \widehat{A}$,

$$
\chi_{\lambda}(\widehat{A N} R)(x)= \begin{cases}r^{m}, & x=1, \\ r^{m / 2}, & x \in h k\left\langle h^{2}\right\rangle, \\ -1, & x \in h\left\langle k, h^{2}\right\rangle C \backslash h k\left\langle h^{2}\right\rangle, \\ 1, & x \in\left(\left\langle k, h^{2}\right\rangle C\right)^{\#} .\end{cases}
$$


(2) $\widehat{A}=\left\langle h, k, i: h^{8}=k^{2}=-1, i^{4}=1, h^{k}=-h^{-1}, h^{i}=-h^{3},[k, i]=1\right\rangle$, $r=3, m=4$, and $\left\langle h^{2}, h k\right\rangle<A_{0}$. For $x \in \hat{A}$,

$$
\chi_{\lambda}(\widehat{A N R})(x)= \begin{cases}81, & x=1, \\ 9, & x \in h k\left\langle h^{2}\right\rangle \cup\left\{i^{2},-i^{2}\right\}, \\ -3, & x \in\left\{i, i^{-1}\right\}\left(\left\langle h^{2}\right\rangle \cup k\left\langle h^{4}\right\rangle\right), \\ -1, & x \in h\left\langle h^{2}, k i\right\rangle, \\ 1, & \text { otherwise. }\end{cases}
$$

(3) $\widehat{A}=\left\langle h, k, j: h^{2^{s}}=k^{2}=-1, j^{2}=1, h^{k}=-h^{-1}, h^{j}=-h,[k, j]=1\right\rangle$, $r=2^{s}+1, m=2$, and $\left\langle h^{2}, h k\right\rangle<A_{0}$. For $x \in \widehat{A}$,

$$
\chi_{\lambda}(\widehat{A N R})(x)= \begin{cases}r^{2}, & x=1, \\ r, & x \in h k\left\langle h^{2}\right\rangle \cup\{j,-j\}, \\ -1, & x \in h\left\langle j, h^{2}\right\rangle, \\ 1, & \text { otherwise. }\end{cases}
$$

(4) $\hat{A}=\langle h, k, j\rangle$ as in (3), $r=2^{s}-1, m=2$, and $\left\langle h^{2}, h k\right\rangle \leq A_{0}$. For $x \in \widehat{A}$,

$$
\chi_{\lambda}(\widehat{A N R})(x)= \begin{cases}r^{2}, & x=1, \\ -r, & x \in h k\left\langle h^{2}\right\rangle \cup\{j,-j\}, \\ -1, & x \in h\left\langle j, h^{2}\right\rangle, \\ 1, & \text { otherwise. }\end{cases}
$$

Remarks. Before proving the theorem we want to explicitly note some of the similarities between these characters. In all cases $m$ is even. Also, $1 \neq|N|$ divides $r^{m / 2} \pm 1$ and so $r^{m / 2} \geq 5$. Notice that $\chi_{\lambda}(x)= \pm r^{m / 2}$ iff $x$ is a noncentral involution and that $\chi_{\lambda}(x)=1$ for $1 \neq x \in\left\langle h^{2}\right\rangle=\widehat{A}^{\prime}$. These facts will be useful in $\S 6$.

Proof. Set $\chi_{\lambda}=\chi_{\lambda}(\widehat{A} N R)$. Since $\left.\chi_{\lambda}\right|_{A_{0}} \nsucceq \rho_{A_{0}}$, clearly $\left.\chi_{\lambda}\right|_{\widehat{A}} \geq \rho_{\widehat{A}}$ and $\widehat{A}, N$, and $R$ are one of the entries (3) or (4), (10), (11), or (12) of Table (3.14). We obtain the groups $\hat{A}$ using Examples (3.10) and (3.12). Let $\mu$ be the 2-part of $\nu$. Then $\mu \in\langle\nu\rangle$ and $|\mu|=|\nu|_{2}$. We then obtain the groups $\hat{A}$ using the following correspondences.

(1) $h=\left[\begin{array}{ll}\mu & \\ & \mu^{-1}\end{array}\right], \quad k=\left[\begin{array}{ll} & \psi \\ -\psi & \end{array}\right]$,

(2) $h=\left[\begin{array}{ll}\mu & \\ & \mu^{-1}\end{array}\right], \quad k=\left[\begin{array}{ll}\sigma^{2} & \sigma^{2}\end{array}\right], \quad i=\left[\begin{array}{ll}\sigma & \\ & \sigma\end{array}\right]$,

(3) $h=\left[\begin{array}{ll}\mu & \\ & \mu^{-1}\end{array}\right], \quad k=\left[\begin{array}{cc} & \sigma \\ -\sigma & \end{array}\right], \quad j=\left[\begin{array}{ll}\sigma & \\ & \sigma\end{array}\right]$,

(4) $h=\left[\begin{array}{ll}\mu & \\ & \mu^{-1}\end{array}\right], \quad k=\left[\begin{array}{ll}\sigma \mu & \\ & \sigma \mu^{-1}\end{array}\right], \quad j=\left[\begin{array}{ll} & \sigma \mu^{2^{s-1}} \\ -\sigma \mu^{2^{s-1}} & \end{array}\right]$. 
The character values of $\chi_{\lambda}$ are computed using Theorem (2.3). First consider $x \in A^{\#}$ with $\langle x\rangle \cap Z(A) \neq 1$. Then $\left.\bar{R}\right|_{\langle x\rangle}=\bar{R}_{\langle 1\rangle}$ is a sum of faithful modules. Since in each example $r^{m} \equiv 1(\bmod |h|)$, where $|h|=2^{s+1} \| r^{m}-1$, we have $\chi_{\lambda}(x)=-1$ if $|x|_{2}=|h|$ and $\chi_{\lambda}(x)=1$ if $|x|_{2}<|h|$.

Suppose next that $x$ is a noncentral involution. Then $\left.\bar{R}\right|_{\langle x\rangle}=\bar{R}_{\langle x\rangle} \dot{+} \bar{R}_{\langle 1\rangle}$, where by Lemma (2.4) both $\bar{R}_{\langle x\rangle}=C_{\bar{R}}(x)$ and $\bar{R}_{\langle 1\rangle}=[\bar{R}, x]$ have dimension $r^{m / 2}$. Now $r^{m / 2} \equiv 1(\bmod 2)$ and so we can take $\varepsilon_{\langle 1\rangle}=1$. In (1), (2), and (3) $\left(r^{m / 2}-1\right) / 2$ is even and so $\chi_{\lambda}(x)=\varepsilon_{\langle 1\rangle} \gamma_{\langle 1\rangle} r^{m / 2}=r^{m / 2}$. In (4) $\left(r^{m / 2}-1\right) / 2$ is odd and so $\gamma_{\langle 1\rangle}=-1$ and $\chi_{\lambda}(x)=-r^{m / 2}$.

All elements of the groups in (1), (3), and (4) fall into one of the two categories above. However, the group in (2) also contains elements $x$ of order 4 for which $\langle x\rangle \cap Z(A)=1$. For these elements $\left.\bar{R}\right|_{\langle x\rangle}=\bar{R}_{\langle x\rangle} \dot{+} \bar{R}_{\left\langle x^{2}\right\rangle}+\bar{R}_{\langle 1\rangle}$ and by (2.4) $\operatorname{dim} \bar{R}_{\langle x\rangle}=\operatorname{dim} \bar{R}_{\left\langle x^{2}\right\rangle}=1$ and $\operatorname{dim} \bar{R}_{\langle 1\rangle}=2$. Now since $r=3$ and $\left|\bar{R}_{\langle 1\rangle}\right|=9 \equiv 1(\bmod 4)$ and $(9-1) / 4$ is even, we have $\varepsilon_{\langle 1\rangle}=\gamma_{\langle 1\rangle}=1$. Also, $\left|\bar{R}_{\langle x\rangle}\right|=3 \equiv 1(\bmod 2)$ and $(3-1) / 2$ is odd and so $\varepsilon_{\left\langle x^{2}\right\rangle}=1$ while $\gamma_{\left\langle x^{2}\right\rangle}=-1$. Then $\chi_{\lambda}(x)=3 \cdot 1 \cdot(-1) \cdot 1 \cdot 1=-3$.

Using the character values, in each case a routine calculation shows that for every nonlinear irreducible $\widehat{A}$-character $\mu,\left(\left.\chi_{\lambda}\right|_{\widehat{A}}, \mu\right) \geq \mu(1) / 2$. Then $\left.\chi_{\lambda}\right|_{\widehat{A}} \geq$ $\left(\rho_{\widehat{A}}-\rho_{\widehat{A} / \widehat{A}^{\prime}}\right) / 2$.

To complete the proof we must determine the subgroups $B \leq \widehat{A}$ for which $\left.\chi_{\lambda}\right|_{B} \nsucceq \rho_{B}$, and the $B$ 's for which $\left.\chi_{\lambda}\right|_{B} \geq \rho_{B}$. We divide the subgroups $B$ of $\widehat{A}$ into three categories:

(1) $\left\langle h^{2}, h k\right\rangle \leq B$,

(2) $\left\langle h^{2}, h k\right\rangle \notin B\left\langle h^{2}\right\rangle$, and

(3) $\left\langle h^{2}, h k\right\rangle \not B$ but $\left\langle h^{2}, h k\right\rangle \leq B\left\langle h^{2}\right\rangle$.

In each case we explicitly give the required character decompositions.

Case 1. $\left\langle h^{2}, h k\right\rangle \leq B$.

Set $D=\left\langle h^{2}, h \bar{k}\right\rangle$. Let $\alpha$ be the linear $D$-character with $\operatorname{ker} \alpha=\left\langle h^{2}\right\rangle$. In exceptions (1), (2), and (3)

$$
\left.\chi_{\lambda}\right|_{D}=\rho_{D}+\left[\left(r^{m / 2}-1\right) / 2\right]\left(\rho_{D}-\alpha\right)+\left[\left(r^{m / 2}+1\right) / 2\right] 1_{D} .
$$

Then $\left.\chi_{\lambda}\right|_{D}$ contains $\rho_{D}$ but not $2 \rho_{D}$. Hence if $D<B$, then $\left.\chi_{\lambda}\right|_{B} \nsupseteq \rho_{B}$. In exception (4)

$$
\left.\chi_{\lambda}\right|_{D}=[(r-1) / 2]\left(\rho_{D}-1_{D}\right)+[(r+1) / 2] \alpha .
$$

Thus, $\left.\chi_{\lambda}\right|_{D} \nsupseteq \rho_{D}$ and hence, if $D \leq B$, then $\left.\chi_{\lambda}\right|_{B} \nsucceq \rho_{B}$.

Case 2. $\left\langle h^{2}, h k\right\rangle \not B B\left\langle h^{2}\right\rangle<\hat{A}$.

We will show that $\left.\chi_{\lambda}\right|_{B} \geq \rho_{B}$ for each such subgroup $B$. In each example $\widehat{A} /\left\langle h^{2}\right\rangle$ is abelian with $\left\langle h^{2}, h k\right\rangle /\left\langle h^{2}\right\rangle$ as a direct summand. Then $B \leq \widehat{B}$, where

$$
\widehat{A} /\left\langle h^{2}\right\rangle=\left\langle h^{2}, h k\right\rangle /\left\langle h^{2}\right\rangle+\widehat{B} /\left\langle h^{2}\right\rangle .
$$

Then $\left\langle h^{2}, h k\right\rangle \not \widehat{B}$ and $[\widehat{A}: \widehat{B}]=2$. Since $\left.\chi_{\lambda}\right|_{\widehat{B}} \geq \rho_{\widehat{B}}$ implies $\left.\chi_{\lambda}\right|_{B} \geq \rho_{B}$, it suffices to consider only those subgroups $B$ which have index 2 in $\hat{A}$. We explicitly list the possibilities for $B$ that occur in each of the four examples. In each case we can write $\left.\chi_{\lambda}\right|_{B}$ in the form $n \rho_{B}+\psi$, as at the end of $\S 1$, and hence $\left.\chi_{\lambda}\right|_{B} \geq \rho_{B}$. 
Case 2a. $\widehat{A}=C \times\langle h, k\rangle$ is the group of (1).

Here $B=\langle h\rangle \times C$ or $\left\langle h^{2}, k\right\rangle \times C$. We have

$$
\left.\chi_{\lambda}\right|_{B}=\left[\left(r^{m / 2}+1\right) / 2|C|\right] \rho_{B}+\mu
$$

where in the first case $\mu$ is the linear character with $\operatorname{ker} \mu=\left\langle h^{2}\right\rangle \times C$ and in the second $\mu=1_{B}$.

Case 2b. $\widehat{A}=\langle h, k, i\rangle$ is the group of (2).

Here $B$ is one of $\langle h, i\rangle,\langle h, k i\rangle,\left\langle h^{2}, k, i\right\rangle$, or $\left\langle h^{2}, k, h i\right\rangle$. Set $I=$ $\left\langle-1, i^{2}\right\rangle, J=\left\langle i^{2}\right\rangle B^{\prime}$, and

$$
\varphi=\rho_{B}+\frac{1}{4}\left(\rho_{B}-\rho_{B /\langle-1\rangle}\right)+\frac{1}{2}\left(\rho_{B / I}-\rho_{B / J}\right) .
$$

When $B=\langle h, i\rangle$,

$$
\left.\chi_{\lambda}\right|_{B}=\varphi+\mu_{1}+2 \mu_{2},
$$

where $\operatorname{ker} \mu_{1}=\left\langle h, i^{2}\right\rangle$ and $\operatorname{ker} \mu_{2}=\left\langle h^{2}, h i\right\rangle$. When $B=\langle h, k i\rangle$,

$$
\left.\chi_{\lambda}\right|_{B}=\varphi+\left(\rho_{B / J}-\rho_{B /\langle k i\rangle B^{\prime}}\right)+\mu,
$$

where $\operatorname{ker} \mu=\left\langle k i, h^{2}\right\rangle$. When $B=\left\langle h^{2}, k, i\right\rangle$.

$$
\left.\chi_{\lambda}\right|_{B}=\varphi+\left(\rho_{B / J}-\rho_{B /\langle k i\rangle B^{\prime}}-\mu_{1}\right)+\mu_{2}+\mu_{3},
$$

where $\operatorname{ker} \mu_{1}=\left\langle h^{2}, i\right\rangle, \operatorname{ker} \mu_{2}=\left\langle h^{2}, k, i^{2}\right\rangle$, and $\operatorname{ker} \mu_{3}=\left\langle h^{2}, k i\right\rangle$. When $B=\left\langle h^{2}, k, h i\right\rangle$,

$$
\left.\chi_{\lambda}\right|_{B}=\varphi+\left(\rho_{B / J}-\rho_{B /\langle h k i\rangle B^{\prime}}\right)+1_{B} .
$$

Case 2c. $\widehat{A}=\langle h, k, j\rangle$ is the group of (3) and (4).

Here $B$ is one of $\langle h, j\rangle,\left\langle h^{2}, k, j\right\rangle,\langle h, k j\rangle$, or $\left\langle h^{2}, k, h j\right\rangle$. Let $\varepsilon \equiv r^{m / 2}$ $(\bmod 4)$. Set $H=\left\langle h^{2}\right\rangle$ and

$$
\varphi=[(r-2+\varepsilon) / 4] \rho_{B}+\frac{1}{2}\left(\rho_{B}-\rho_{B / H}\right) .
$$

Suppose $j \in B$. Set $\varphi_{1}=\rho_{B /\langle-1, j\rangle}$ and $\varphi_{-1}=\rho_{B /\langle-1\rangle}-\rho_{B /\langle-1, j\rangle}$. Then

$$
\left.\chi_{\lambda}\right|_{B}=\varphi+\varphi_{\varepsilon}+\mu,
$$

where $\operatorname{ker} \mu=\left\langle h^{2}, j\right\rangle$ when $B=\langle h, j\rangle$ and $\mu=1_{B}$ when $B=\left\langle h^{2}, k, j\right\rangle$. When $j \notin B$,

$$
\left.\chi_{\lambda}\right|_{B}=\varphi+\left(\rho_{B / H}-\mu\right),
$$

where $\operatorname{ker} \mu=\langle h\rangle$ when $B=\langle h, k j\rangle$ and $\operatorname{ker} \mu=\left\langle h^{2}, h j\right\rangle$ when $B=$ $\left\langle h^{2}, k, h j\right\rangle$.

Case 3. $\left\langle h^{2}, h k\right\rangle \not B B$ but $\left\langle h^{2}, h k\right\rangle \leq B\left\langle h^{2}\right\rangle$.

Again we show that $\left.\chi_{\lambda}\right|_{B} \geq \rho_{B}$ always and so, as above, it suffices to consider only those groups $B$ which are maximal with respect to the conditions given. Now $h^{2 n+1} k \in B$, some $n$. Since all such elements are conjugate we may assume $h k \in B$. Then $h^{2} \notin B$. Since $h^{2} \notin B$ implies $h^{2} \notin\left\langle h^{4}\right\rangle B$ we may assume that $B=\left\langle h^{4}\right\rangle B$, i.e., $h^{4} \in B$. Now

$$
B^{\prime} \leq B \cap \widehat{A^{\prime}}=B \cap\left\langle h^{2}\right\rangle=\left\langle h^{4}\right\rangle
$$

and so $\left\langle h^{4}, h k\right\rangle \triangleleft B$ and $B \leq N_{\widehat{A}}\left(\left\langle h^{4}, h k\right\rangle\right)$.

Case 3a. $\widehat{A}=C \times\langle h, k\rangle$ is the group of (1). 
Here $B \leq N_{\widehat{A}}\left(\left\langle h^{4}, h k\right\rangle\right)=C \times\left\langle h^{2}, h k\right\rangle$ and we may assume that $B=$ $C \times\left\langle h^{4}, h k\right\rangle$. Then

$$
\left.\chi_{\lambda}\right|_{B}=\left[\left(r^{m / 2}+1\right) / 2|C|\right]\left[\rho_{B}+\left(\rho_{B}-\rho_{B /\left\langle h^{2}\right\rangle}\right)+2 \rho_{C}\right]+\mu,
$$

where $\operatorname{ker} \mu=\left\langle h^{4}\right\rangle C$.

Case 3b. $\hat{A}$ is the group of (2), (3), or (4).

When $\widehat{A}$ is the group of (2), set $j=i^{2}$. Then, in all cases, $B \leq N_{\widehat{A}}\left(\left\langle h^{4}, h k\right\rangle\right)$ $=\left\langle h^{2}, h k, j\right\rangle$ and $B$ is either $\left\langle h^{4}, h k, h^{2} j\right\rangle$ or $\left\langle h^{4}, h k, j\right\rangle$. Let $\varepsilon \equiv r^{m / 2}$ $(\bmod 4)$ and set $H=\left\langle h^{4}\right\rangle$.

Suppose first that $B=\left\langle h^{2}, h k, h^{2} j\right\rangle$. Set $\varphi_{1}=\rho_{B / H\langle h k\rangle}$ and $\varphi_{-1}=\rho_{B / H}-$ $\rho_{B / H\langle h k\rangle}$. Then

$$
\begin{aligned}
\left.\chi_{\lambda}\right|_{B}= & {\left[\left(r^{m / 2}+3 \varepsilon\right) / 4\right] \rho_{B}+\left[\left(r^{m / 2}-\varepsilon\right) / 4\right]\left(\rho_{B}-\rho_{B / H}\right) } \\
& +\left[\left(r^{m / 2}-\varepsilon\right) / 2\right] \varphi_{\varepsilon}+1_{B} .
\end{aligned}
$$

Now suppose that $B=\left\langle h^{4}, h k, j\right\rangle$. Set $\varphi_{1}=\rho_{B /\langle-1, j\rangle}, \varphi_{-1}=\rho_{B /\langle-1\rangle}-$ $\rho_{B /\langle-1, j\rangle}, \mu_{1}=1_{B}$, and let $\mu_{-1}$ be the linear character with kernel $\left\langle h^{4}, h k\right\rangle$. Then

$$
\begin{aligned}
\left.\chi_{\lambda}\right|_{B}= & {\left[\left(r^{m / 2}-2+\varepsilon\right) / 4\right] \rho_{B}+\left[\left(r^{m / 2}+2+\varepsilon\right) / 4\right]\left(\rho_{B}-\rho_{B /\langle-1\rangle}\right) } \\
& +\left[\left(r^{m / 2}-2+\varepsilon\right) / 4\right]\left(\rho_{B /\langle-1\rangle}-\rho_{B / H}\right)+2 \varphi_{\varepsilon} \\
& +\left[\left(r^{m / 2}-1\right) / 2\right] \varphi_{\varepsilon}+\varepsilon \mu_{\varepsilon} .
\end{aligned}
$$

\section{TENSOR INDUCED MODULES AND CHARACTERS}

Tensor induction of modules is similar to ordinary induction. However, where in ordinary induction you take the direct sum of modules, here we take the tensor product. The analysis here is similar to that of $[5, \S 2 ; 14, \S 3]$.

Throughout this section we will have $H$ a subgroup of a group $G, k$ a field, $U$ a $k[H]$-module, and $\chi$ the character of $H$ on $U$. We wish to describe the action of $G$ on $V=\left.U\right|^{\otimes G}$ and the character $\left.\chi\right|^{\otimes G}$. Let $T=\left\{y_{1}, y_{2}, \ldots, y_{n}\right\}$ be a transversal for $H$ in $G$. Set

$$
V=\left(y_{1} \otimes U\right) \otimes\left(y_{2} \otimes U\right) \otimes \cdots \otimes\left(y_{n} \otimes U\right) .
$$

Here the tensor in $\left(y_{i} \otimes U\right)$ is over $k[H]$ and the tensors between the $\left(y_{i} \otimes U\right)$ 's are over $k$. For $g \in G, g y_{i} H=y_{j} H$, some $j$. Define a map $g \rightarrow \hat{g}$ from $G$ into $S_{n}$, the symmetric group on $n$ letters, by $\hat{g}(i)=j$ iff $g y_{i} H=y_{j} H$, or equivalently, iff $y_{j}^{-1} g y_{i} \in H$. It is easily verified that this is a homomorphism. Define a map from $G$ into $H^{(n)}=\left\{\left(h_{1}, h_{2}, \ldots, h_{n}\right): h_{i} \in H\right\}$ by

$$
g \rightarrow F_{g}=\left(y_{\hat{g}(1)}^{-1} g y_{1}, y_{\hat{g}(2)}^{-1} g y_{2}, \ldots, y_{\hat{g}(n)}^{-1} g y_{n}\right) \text {. }
$$

In ordinary induction we have $g\left(y_{i} \otimes u\right)=y_{\hat{g}(i)} \otimes y_{\hat{g}(i)}^{-1} g y_{i} u$ and for $v=\left(y_{1} \otimes\right.$ $\left.u_{1}\right)+\left(y_{2} \otimes u_{2}\right)+\cdots+\left.\left(y_{n} \otimes u_{n}\right) \in U\right|^{G}$ we have

$$
\begin{aligned}
g v & =\left(y_{\hat{g}(1)} \otimes y_{\hat{g}(1)}^{-1} g y_{1} u_{1}\right)+\cdots+\left(y_{\hat{g}(n)} \otimes y_{\hat{g}(n)}^{-1} g y_{n} u_{n}\right) \\
& =\left(y_{\hat{g}(1)} \otimes F_{g}(1) u_{1}\right)+\cdots+\left(y_{\hat{g}(n)} \otimes F_{g}(n) u_{n}\right) \\
& =\left(y_{1} \otimes F\left(\hat{g}^{-1}(1)\right) u_{\hat{g}^{-1}(1)}\right)+\cdots+\left(y_{n} \otimes F\left(\hat{g}^{-1}(n)\right) u_{\hat{g}^{-1}(n)}\right) .
\end{aligned}
$$


In tensor induction the equation $g\left(y_{i} \otimes u\right)=y_{\hat{g}(i)} \otimes y_{\hat{g}(i)}^{-1} g y_{i} u$ describes the affect of $g$ on a factor in a tensor of $\left.U\right|^{\otimes G}$. That is, for $g \in G$ and

$$
v=\left.\left(y_{1} \otimes u_{1}\right) \otimes\left(y_{2} \otimes u_{2}\right) \otimes \cdots \otimes\left(y_{n} \otimes u_{n}\right) \in U\right|^{\otimes G},
$$

we have

$$
g v=\left(y_{1} \otimes F\left(\hat{g}^{-1}(1)\right) u_{\hat{g}^{-1}(1)}\right) \otimes \cdots \otimes\left(y_{n} \otimes F\left(\hat{g}^{-1}(n)\right) u_{\hat{g}^{-1}(n)}\right) .
$$

Let $\widehat{G}$ be the image of $G$ in $S_{n}$ under the map $g \rightarrow \hat{g}$. Define

$$
\varphi: G \rightarrow H \sim \widehat{G} \quad \text { by } \varphi(g)=\hat{g} F_{g} \text {. }
$$

Straightforward computation shows that $\varphi$ is a monomorphism. Recall that $U^{(n)}$ is an $(H \sim \widehat{G})$-module with the following action. For $\left(h_{1}, \ldots, h_{n}\right) \in H^{(n)}$, $\hat{g} \in \widehat{G}$, and $u_{1} \otimes \cdots \otimes u_{n} \in U^{(n)}$,

$$
\begin{gathered}
\hat{g}\left(h_{1}, \ldots, h_{n}\right)\left(u_{1} \otimes \cdots \otimes u_{n}\right)=\hat{g}\left(h_{1} u_{1} \otimes \cdots \otimes h_{n} u_{n}\right) \\
=h_{\hat{g}^{-1}(1)} u_{\hat{g}^{-1}(1)} \otimes \cdots \otimes h_{\hat{g}^{-1}(n)} u_{\hat{g}^{-1}(n)} .
\end{gathered}
$$

That is, $\left(h_{1}, h_{2}, \ldots, h_{n}\right)$ acts componentwise and $\hat{g}$ moves the $i$ th factor to the $\hat{g}(i)$ th position.

Define

$$
I:\left.U\right|^{\otimes G} \rightarrow U^{(n)} \quad \text { by } I\left(\left(y_{1} \otimes u_{1}\right) \otimes \cdots \otimes\left(y_{n} \otimes u_{n}\right)\right)=u_{1} \otimes \cdots \otimes u_{n} .
$$

Clearly $I$ is an isomorphism. From the computations above it is clear that for $g \in G$ and $\left.v \in U\right|^{\otimes G}, I(g v)=\varphi(g) I(v)$. Let $H_{0}=C_{H}(U)$ and $\bar{H}=H / H_{0}$. Now $H_{0}^{(n)} \leq C_{H \sim \widehat{G}^{\prime}}\left(U^{(n)}\right)$ and $U^{(n)}$ is naturally an $(H \sim \widehat{G}) / H_{0}^{(n)} \simeq \bar{H} \sim \widehat{G}$ module. Let $\bar{\varphi}$ be the composition map

$$
\bar{\varphi}: G \rightarrow H \sim \widehat{G} \rightarrow \bar{H} \sim \widehat{G} .
$$

Then $\operatorname{ker} \bar{\varphi} \leq C_{G}\left(\left.U\right|^{\otimes G}\right.$ and $G / \operatorname{ker} \bar{\varphi}$ acts on $\left.U\right|^{\otimes G}$ as a subgroup of $\bar{H} \sim \widehat{G}$ acting on $U^{(n)}$. In summary, we have

(4.1) Lemma. Let $G$ be a group, $H$ a subgroup of index $n, k$ a field, and $U$ a $k[H]$-module. Fix a transversal $T$ for $H$ in $G$. Let $\varphi: G \rightarrow H \sim \widehat{G}$, $\bar{\varphi}: G \rightarrow \bar{H} \sim \widehat{G}$, and $I:\left.U\right|^{\otimes G} \rightarrow U^{(n)}$ be the maps $(\varphi),(I)$, and $(\bar{\varphi})$ above. Then for $g \in G$ and $\left.v \in U\right|^{\otimes G}, I(g v)=\varphi(g) I(v)=\bar{\varphi}(g) I(v)$. Also, $\operatorname{ker} \bar{\varphi} \leq$ $C_{G}\left(\left.U\right|^{\otimes G}\right)$ and so $G / \operatorname{ker} \bar{\varphi}$ acts on $\left.U\right|^{\otimes G}$ as a subgroup of $\bar{H} \sim \widehat{G}$ acting on $U^{(n)}$. Further, $\varphi(G)$ and $\bar{\varphi}(G)$ cover $\widehat{G}$.

Suppose now that $U$ is in fact a $G$-module and that $G=H C_{G}(U)$. Let $V=\left.\left.U\right|_{H}\right|^{\otimes G}$. We explicitly describe $\bar{\varphi}(G)$. Since $G=H C_{G}(U)$, we can choose the transversal $T$ so that each $y_{i} \in C_{G}(U)$. Let $g \in G$. Then $g=y_{i} h$ for some $h \in H$ and some $i$. Then for $u \in U, y_{\hat{g}(i)}^{-1} g y_{i} u=g u=y_{i} h u=h u$. Then $\varphi(g) \equiv \hat{g}(h, h, \ldots, h) \bmod H_{0}^{(n)}$. So, $\bar{\varphi}(g)=\hat{g}(\bar{h}, \bar{h}, \ldots, \bar{h}) \in \bar{H}^{\Delta} \times \widehat{G}$. Thus, we have

(4.2) Lemma. Assume that $U$ is a $G$-module and $G=H C_{G}(U)$, and set $V=$ $\left.\left.U\right|_{H}\right|^{\otimes G}$. Choose the transversal $T$ so that $T \subseteq C_{G}(U)$. Then $\bar{\varphi}: G \rightarrow \bar{H}^{\Delta} \times \widehat{G}$ and so $G / \operatorname{ker} \bar{\varphi}$ acts on $V$ as a subgroup of $\bar{H}^{\Delta} \times \widehat{G}$ acting on $U^{(n)}$.

We will in general be applying these lemmas when $G$ is nilpotent and $H \triangleleft G$ of prime index $p$. We can then choose $y \in G \backslash H$ of $p$-power order and let $T=$ 
$\left\{1, y, y^{2}, \ldots, y^{p-1}\right\}$. Then $\widehat{G}=\langle(12 \cdots p)\rangle \simeq Z_{p}$. Further, for $g \in \mathscr{O}_{p^{\prime}}(G)$, $y g=g y$ and so $\hat{g}=1$ and $F_{g}=(g, g, \ldots, g)$. Then we have

(4.3) Lemma. Assume $G$ is nilpotent and $H \triangleleft G$ of prime index $p$. Then $\widehat{G} \simeq Z_{p}$ and $\bar{\varphi}(G) \leq \mathscr{O}_{p^{\prime}}(\bar{H})^{\Delta} \times\left(\mathscr{O}_{p}(\bar{H}) \sim Z_{p}\right)$.

We can modify the argument to obtain

(4.4) Lemma. Assume $G$ is nilpotent and $[G: H]$ is a p-power. Then $\widehat{G}$ is a p-group and $\bar{\varphi}(G) \leq \mathscr{O}_{p^{\prime}}(\bar{H})^{\Delta} \times\left(\mathscr{O}_{p}(\bar{H}) \sim \widehat{G}\right)$. In particular, if $\left.U\right|^{\otimes G}$ is a faithful $G$-module, then $\mathscr{O}_{p^{\prime}}(G) \simeq \mathscr{O}_{p^{\prime}}(\bar{H})$.

We next look at the characters of induced modules. Set $n=[G: H]$. Let $\chi$ be the character of $H$ on $U$. Then $\left.\chi\right|^{\otimes G}$ denotes the character of $G$ on $\left.U\right|^{\otimes G}$ and $\chi^{(n)}$ the character of $H \sim \widehat{G}$ on $U^{(n)}$. Since $H_{0}^{(n)} \leq C_{H \sim \widehat{G}}\left(U^{(n)}\right)$, we can view $\chi^{(n)}$ as an $(\bar{H} \sim \widehat{G})$-character. By Lemma (4.1), for $g \in G$, $\left.\chi\right|^{\otimes G}(g)=\chi^{(n)}(\varphi(g))=\chi^{(n)}(\bar{\varphi}(g))$.

(4.5) Lemma [12, 2.1]. Assume $H \triangleleft G$ of prime index $p$. Choose $y \in G \backslash H$. (Then $T=\left\{1, y, y^{2}, \ldots, y^{p-1}\right\}$ is a transversal for $H$ in $G$.) Then

(i) for $g \in G$,

$$
\left.\chi\right|^{\otimes G}(g)= \begin{cases}\prod_{i=0}^{p-1} \chi\left(g^{y^{i}}\right), & g \in H, \\ \chi\left(g^{p}\right), & g \in G \backslash H ;\end{cases}
$$

(ii) for $g=\left(h_{1}, h_{2}, \ldots, h_{p}\right) y \in H \sim Z_{p}=H^{(p)} Z_{p}$,

$$
\chi^{(n)}(g)= \begin{cases}\prod_{i=1}^{p} \chi\left(h_{i}\right), & g \in H^{(p)}, \\ \chi\left(\prod_{i=1}^{p} h_{i}\right)=\chi\left(g^{p}\right), & g \in\left(H \sim Z_{p}\right) \backslash H^{(p)} .\end{cases}
$$

If $U$ is in fact a $k[G]$-module, then $\chi$ is a $G$-character and $\chi\left(g^{y^{i}}\right)=\chi(g)$. Then

(4.6) Corollary. Assume $H \triangleleft G$ of prime index $p$ and that $U$ is a G-module. Then

$$
\left.\left.\chi\right|_{H}\right|^{\otimes G}(g)= \begin{cases}\chi(g)^{p}, & g \in H \\ \chi\left(g^{p}\right), & g \notin H .\end{cases}
$$

\section{THE INDUCTIVE LEMMAS}

The following very important proposition enables us to relate the presence of regular modules in $\left.V_{\lambda}(A M R)\right|_{A}$ in the general case to what happens in the form quasiprimitive case.

(5.1) Proposition [5, 5.18]. Assume $G=A M R$ satisfies Hypothesis (3.5). Set $\bar{R}=R / Z(R)$. By (3.6), $\bar{R}=\left.\bar{R}_{0}\right|^{A M}$, where $\bar{R}_{0}$ is a form primitive $A_{0} M_{0}$ module, $A_{0} \leq A$ and $M_{0} \leq M$. Then

$$
\left.V_{\lambda}(A M R)\right|_{A M} \simeq W \otimes\left(\left.\left.V_{\lambda}\left(A_{0} M_{0} R_{0}\right)\right|_{A_{0} M_{0}}\right|^{\otimes A M}\right),
$$

where $W$ is a linear AM-module.

When the module $\left.V_{\lambda}\left(A_{0} M_{0} R_{0}\right)\right|_{A_{0}}$ does not contain a regular module, we will have enough information to explicitly compute the character $\left.\chi_{\lambda}(A M R)\right|_{A}$ of the module $\left.V_{\lambda}(A M R)\right|_{A}$. We can then determine whether $\left.\chi_{\lambda}(A M R)\right|_{A} \geq 3 \rho_{A}$. 
When the module $\left.V_{\lambda}\left(A_{0} M_{0} R_{0}\right)\right|_{A_{0}}$ does contain copies of the regular module, these can be used to obtain copies of the regular module in $\left.V_{\lambda}(A M R)\right|_{A} \simeq$ $\left.\left.\left.W \otimes V_{\lambda}\left(A_{0} M_{0} R_{0}\right)\right|_{A_{0} M_{0}}\right|^{\otimes A M}\right|_{A}$. The lemmas we will need to do this follow.

In discussing why a module $V=\left.U\right|^{\otimes G}$ might contain regular $G / C_{G}(V)$ modules, many modules arise and it is inconvenient to identify their centralizers. So we make the following definition.

(5.2) Definition. Let $G$ be a group, $V$ a $G$-module, and $\chi$ the character of $V$. We say $V$ contains $n$ regular* $G$-modules if it has a submodule isomorphic to the direct sum of $n$ copies of the regular $G / C_{G}(V)$-module. We denote the character of the regular* module by $\rho_{G}^{*}$ and write $\chi \geq n \rho_{G}^{*}$.

(5.3) Lemma [18, p. 101]. Suppose $V$ is a faithful $G$-module and that $V$ contains $t \geq 2$ regular $G$-modules. Let $p$ be a prime and $Z_{p}=\langle(12 \cdots p)\rangle \leq S_{p}$. Then $V^{(p)}$ contains at least $\left(t^{p}-t\right) / p$ copies of the regular $\left(G \sim Z_{p}\right)$-module.

(5.4) Corollary. Suppose $H \triangleleft G$ of prime index $p$. Let $U$ be an $H$-module and suppose $U$ contains $t \geq 2$ copies of the regular ${ }^{*} H$-module. Then $\left.U\right|^{\otimes G}$ contains at least $\left(t^{p}-t\right) / p$ copies of the regular* $G$-module.

Proof. Let $H_{0}=C_{H}(U), \bar{H}=H / H_{0}$, and $\bar{\varphi}$ be as in $\S 4$. Since $H \triangleleft G$ with prime index $p, \widehat{G} \simeq Z_{p}$. Then $\bar{\varphi}: G \rightarrow \bar{H} \sim Z_{p}$ and $G / \operatorname{ker} \bar{\varphi}$ acts on $\left.U\right|^{\otimes G}$ as a subgroup of $\bar{H} \sim Z_{p}$ acting on $U^{(n)}$. But $U$ contains $t$ copies of the regular $\bar{H}$-module and so $U^{(n)}$ contains $\left(t^{p}-t\right) / p$ copies of the regular $\left(\bar{H} \sim Z_{p}\right)$ module and hence $\left.U\right|^{\otimes G}$ contains $\left(t^{p}-t\right) / p$ copies of the regular $(G / \operatorname{ker} \bar{\varphi})$ module and so of the regular* $G$-module.

Remark. We always have $\operatorname{ker} \bar{\varphi}=\varphi^{-1}\left(H_{0}^{(n)}\right)=\bigcap_{g \in G} H_{0}^{g^{-1}} \leq C_{G}\left(\left.U\right|^{\otimes G}\right)$. When $U^{(n)}$ contains the regular $(\bar{H} \sim \widehat{G})$-module, then $\left.U\right|^{\otimes G}$ contains the regular $(G / \operatorname{ker} \bar{\varphi})$-module and so $\operatorname{ker} \bar{\varphi}=C_{G}\left(\left.U\right|^{\otimes G}\right)$.

Using mathematical induction on $[G: H]$ it is easy to prove

(5.5) Corollary [5, 4.4]. Let $G$ be a nilpotent group, $H$ a subgroup of $G$, and $U$ an $H$-module. Set $V=\left.U\right|^{\otimes G}$. Then

(i) if $U$ contains three copies of the regular* $H$-module, then $V$ contains three copies of the regular* G-module, and

(ii) if $|G|$ is odd and $U$ contains two copies of the regular* $H$-module, then $V$ contains two copies of the regular* G-module.

When $\bar{\varphi}(G)<\bar{H} \sim \widehat{G}$, using various techniques, one can frequently obtain regular* $G$-modules even when $U$ contains only one copy of the regular* $H$ module.

In [5, Theorem 4.4] one of us has shown that if $U$ contains, but does not equal, the regular* $H$-module and $G$ is $Z_{p} \sim Z_{p}$ free for all primes $p$, then $V$ contains, but does not equal, the regular ${ }^{*}$ module. In fact, it is only the "top" of $G$ that needs to be $Z_{p} \sim Z_{p}$ free. With minor modifications of the proof one can obtain the following useful result.

(5.6) Theorem. Let $G$ be a nilpotent group and let $H \triangleleft G$ of prime index $p$. Let $U$ be an $H$-module which contains, but does not equal, the regular ${ }^{*} H$-module. Set $\bar{H}=H / C_{H}(U), V=\left.U\right|^{\otimes G}$, and $\bar{G}=G / C_{G}(V)$. Now $\bar{G}$ acts on $V$ as a 
subgroup of $\mathscr{O}_{p^{\prime}}(\bar{H}) \times\left(\mathscr{O}_{p}(\bar{H}) \sim Z_{p}\right)$ acting on $U^{(p)}$. Assume

$$
\mathscr{O}_{p}(\bar{G})<\mathscr{O}_{p}(\bar{H}) \sim Z_{p} .
$$

Then $V$ contains, but does not equal, the regular* $G$-module.

When applying this we will usually have $\bar{G}=\bar{H} \times Z_{p}$. In this case

$$
\left[\mathscr{O}_{p}(\bar{H}) \sim Z_{p}: \mathscr{O}_{p}(\bar{G})\right]=\left|\mathscr{O}_{p}(\bar{H})\right|^{p-1} p \text {. }
$$

Hence

(5.7) Corollary. Assume $G, H, p, U$, and $V$ are as in (5.6) and that $\bar{G} \simeq$ $\bar{H} \times Z_{p}$. Then $V$ contains at least $\left|\mathscr{O}_{p}(\bar{H})\right|^{p-1} \geq\left|\mathscr{O}_{p}(\bar{H})\right|$ copies of the regular * G-module.

(5.8) Lemma [5, 4.5]. Let $G$ be a nilpotent group, $H \triangleleft G$ of prime index $p$, and $U$ an $H$-module. Suppose $U$ contains the regular ${ }^{*} H$-module and that $B=\mathscr{O}_{p^{\prime}}\left(H / C_{H}(U)\right) \neq 1$. Then $\left.U\right|^{\otimes G}$ contains the regular ${ }^{*} G$-module. In fact, $\left.U\right|^{\otimes G}$ contains three copies of the regular* $G$-module unless $U$ does not contain two copies of the regular* $H$-module and either

(i) $p=3$ and $|B|=2$, or

(ii) $p=2$ and $|B|=3$ or 5 .

(5.9) Lemma [5, 1.2]. Let $G$ be a group and let $U_{1}, U_{2}, \ldots, U_{n}$ be $G$-modules. Suppose $U_{i}$ contains $t_{i}$ copies of the regular ${ }^{*} G$-module. Set $V=U_{1} \otimes U_{2} \otimes$ $\cdots \otimes U_{n}$, which is also a $\left(G \simeq G^{\Delta} \leq G^{(n)}\right)$-module. Then $V$ contains $\prod t_{i}$ copies of the regular* $G$-module.

(5.10) Lemma. Let $G$ be a group and let $V$ and $W$ be G-modules. Suppose $V$ contains the regular $G$-module. Then $V \otimes W$ contains at least $\operatorname{dim} W$ copies of the regular $G$-module.

Proof. Let $v \in V$ generate a basis for the regular $G$-module and let $\left\{w_{1}, w_{2}\right.$, $\left.\ldots, w_{n}\right\}, n=\operatorname{dim} W$, be a basis for $W$. Then $\left\{g\left(v \otimes w_{i}\right): g \in G, 1 \leq i \leq n\right\}$ is linearly independent. Then $v \otimes w_{1}, \ldots, v \otimes w_{n}$ generate bases for $n=\operatorname{dim} W$ copies of the regular $G$-module in $V \otimes W$.

(5.11) Lemma [14, 4.9]. Let $G \triangleleft A G$, where $(|A|,|G|)=1$. Let $A_{0} G_{0} \leq A G$ with $A_{0} \leq A$ and $G_{0} \leq G$. Then from each double $\left(A, A_{0} G_{0}\right)$-coset of $A G$ we may choose an element $y \in G$ so that

$$
\left(A_{0} G_{0}\right)^{y^{-1}} \cap A=C_{A_{0}}(y) .
$$

(5.12) Lemma [5, 2.9]. Let $G \triangleleft A G$, where $(|A|,|G|)=1$. Let $A_{0} G_{0} \leq A G$ with $A_{0} \leq A$ and $G_{0} \leq G$. Let $U$ be an $A_{0} G_{0}$-module and set $V=\left.U\right|^{\otimes A G}$. Then

$$
\left.V\right|_{A}=\left.\left.U\right|^{\otimes A G}\right|_{A} \simeq \prod_{x}^{\otimes}\left(\left.\left.U\right|_{C_{A_{0}}(U)}\right|^{\otimes A}\right),
$$

where the $x$ 's are appropriately chosen $\left(A, A_{0} G_{0}\right)$-double coset representatives.

\section{THE MAIN RESUlTS}

We now have the background needed to attack the main theorem. We first outline the general steps involved. 
In this case, $\left.\left.\left.V_{\lambda}(A M R)\right|_{A} \simeq W \otimes V_{\lambda}\left(A_{0} M_{0} R_{0}\right)\right|^{\otimes A M}\right|_{A}$, where $W$ is a linear $A$-module, $R_{0} / Z(R)$ is a form quasiprimitive $A_{0} M_{0}$-module, and $1 \neq$ $M_{0} / C_{M_{0}}\left(R_{0}\right)$ is cyclic. We may assume $A_{0} M_{0} / C_{A_{0} M_{0}}\left(R_{0}\right)$ is one of the exceptional cases of Theorem (3.13). In these, $A_{0} / C_{A_{0}}\left(R_{0}\right)$ is essentially a 2-group.

We first look at what happens when inducing through an odd prime dividing [ $\left.A: A_{0}\right]$ and conclude that $\left[A: A_{0}\right]$ must be a power of 2 .

Next, if $M_{0}<M$, then $\left.\left.V_{\lambda}\left(A_{0} M_{0} R_{0}\right)\right|^{\otimes A_{0} M}\right|_{A_{0}}$ is the tensor product of modules of the form $\left.\left.V_{\lambda}\left(A_{0} M_{0} R_{0}\right)\right|_{A_{1}}\right|^{\otimes A_{0}}$, where $A_{1} \leq A_{0}$, so that these modules are investigated. We conclude that $M_{0}=M$.

Concluding the proof is then routine.

In order to keep the proof conceptually manageable, we in essence isolate the major steps of the proof as separate theorems. Throughout, $\lambda \neq 1$ is a linear $Z(R)$-character and $V_{\lambda}$ and $\chi_{\lambda}$ are the module and character of (2.2).

(6.1) Hypothesis. Let $R \triangleleft A N R$ and $N \triangleleft A N$, where $R$ is an extraspecial group of order $r^{2 m+1}, r$ is a prime, $A$ is nilpotent, and $(|A|, r|N|)=(|N|, r)=1$. Suppose $Z(R) \leq Z(A N R)$ and $\bar{R}=R / Z(R)$ is a form quasiprimitive (not necessarily faithful) $A N$-module. Assume $1 \neq N / C_{N}(\bar{R})$ is cyclic.

(6.2) Theorem. Assume Hypothesis (6.1). Let $A_{0} \leq A$ and let $B$ be another nilpotent group with $A_{0} \leq B . \quad(A \leq B$ is possible but not necessary.) Suppose $(|B|, r|N|)=1$ and that $\left[B: A_{0}\right]$ is divisible by an odd prime $p$. Then $\left.\left.V_{\lambda}(A N R)\right|_{A_{0}}\right|^{\otimes B}$ contains three copies of the regular* $B$-module unless $r^{m}=$ $2 p^{a}-1$ for some positive integer $a,\left|N / C_{N}(\bar{R})\right|=2$, and $B / C_{B}\left(\left.\left.V_{\lambda}(A N R)\right|_{A_{0}}\right|^{\otimes B}\right)$ is a p-group. In this exceptional case $A_{0} C_{A}(\bar{R})=A, 1 \neq A / C_{A}(\bar{R})$ is cyclic of order $\left(r^{m}+1\right) / 2$, and $\left.V_{\lambda}(A N R)\right|_{A}$ contains the regular* A-module.

Proof. Set $\bar{A}=A / C_{A}(\bar{R}), \bar{A}_{0}=A_{0} / C_{A_{0}}\left(\bar{R}_{0}\right) \simeq A_{0} C_{A}(\bar{R}) / C_{A}(\bar{R}) \leq \bar{A}$, and $\bar{N}=N / C_{N}(\bar{R})$. Now $C_{A_{0}}(\bar{R}) \leq C_{A_{0}}(\bar{N})$ and so $\bar{A}_{0}$ acts on $\bar{N}$. Then $\bar{A}_{0} \bar{N} \simeq$ $A_{0} N / C_{A_{0} N}(\bar{R})$ and we may view $\chi_{\lambda}=\left.\chi_{\lambda}(A N R)\right|_{A_{0} N}$ as an $\bar{A}_{0} \bar{N}$-character. We assume that $\left.\left.\chi_{\lambda}\right|_{A_{0}}\right|^{\otimes B} \nsucceq 3 \rho_{B}^{*}$. Then by (5.5), $\chi_{\lambda} \nsucceq 3 \rho_{\bar{A}_{0}}$ and so $\left.\chi_{\lambda}\right|_{\bar{A}} \nsucceq 3 \rho_{\bar{A}}$. Hence, by Theorem (3.13) there is a group $\widehat{A}$ with $\bar{A}_{0} \leq \bar{A} \leq \widehat{A}$ such that

Step 1. $\widehat{A}, \bar{N}, R$, and $V_{\lambda}$ are one of the entries of Table (3.14).

Let $C \leq B$ with $A_{0} \triangleleft C$ of index $p$. Set $V=\left.\left.V_{\lambda}\right|_{A_{0}}\right|^{\otimes C}, \bar{C}=C / C_{C}(V)$, and $\psi=\left.\left.\chi_{\lambda}\right|_{A_{0}}\right|^{\otimes C}$, the character of $\bar{C}$ on $V$. By (5.5),

Step 2. $\psi \nsupseteq 3 \rho_{\bar{C}}$.

Suppose $\left.\chi_{\lambda}\right|_{\bar{A}_{0}} \geq \rho_{\bar{A}_{0}}$. Then by (5.8), either (i) $\bar{A}_{0}$ is a $p$-group or (ii) $p=3$, $\left|\mathscr{O}_{3^{\prime}}\left(\bar{A}_{0}\right)\right|=2$, and $\left.\chi_{\lambda}\right|_{\bar{A}_{0}} \nsucceq 2 \rho_{\bar{A}_{0}}$. Inspecting entries of Table (3.14) we see that

Step 3. Either $\bar{A} \bar{N}$ is cyclic or $\left.\chi_{\lambda}\right|_{\bar{A}_{0}} \nsucceq \rho_{\bar{A}_{0}}$.

(If $\chi_{\lambda} \nsucceq \rho_{\widehat{A}}$ and $\left|\bar{A}_{0}\right|=2$, then it follows from (3.15) that $\left.\chi_{\lambda}\right|_{\bar{A}_{0}} \geq 2 \rho_{\bar{A}_{0}}$.)

Case 1. $\bar{A} \bar{N}$ is cyclic.

Here $|\bar{A} \bar{N}|$ divides $r^{m}+1,\left.\chi_{\lambda}\right|_{\bar{A}_{0}}(1)=r^{m}$ by $(2.3)$, and $\left.\chi_{\lambda}\right|_{\bar{A}_{0}}(x)=1$ for $1 \neq x \in \bar{A}_{0}$. Then

$$
\left.\chi_{\lambda}\right|_{\bar{A}_{0}} \geq\left[\left(r^{m}+1\right) /\left|\bar{A}_{0}\right|-1\right] \rho_{\bar{A}_{0}} .
$$

Thus, $\left[\bar{A} \bar{N}: \bar{A}_{0}\right] \leq\left(r^{m}+1\right) /\left|\bar{A}_{0}\right| \leq 3$, and so $|\bar{A}|=\left|\bar{A}_{0}\right|,|\bar{N}|=2$ or 3 , and $\left.\chi_{\lambda}\right|_{\bar{A}_{0}} \geq(|\bar{N}|-1) \rho_{\bar{A}_{0}}$. Since $|\bar{A}|=\left|\bar{A}_{0}\right|$, we have $A=A_{0} C_{A}(\bar{R})$. Suppose 
$|\bar{N}|=3$. Then $\left.\chi_{\lambda}\right|_{\bar{A}_{0}} \geq 2 \rho_{\bar{A}_{0}}$ and $p \geq 5$. Then, by (5.4), $\psi=\left.\left.\chi_{\lambda}\right|_{A_{0}}\right|^{\otimes C} \geq 3 \rho_{C}^{*}$, a contradiction. Then $|\bar{N}|=2$ and so $|B|$ must be odd. If $\bar{A}_{0}$ were not a $p$ group, (5.8) would give $\psi \geq 3 \rho_{C}^{*}$, a contradiction. Thus, $\bar{A}_{0}$ is a $p$-group and $r^{m}+1=\left|\bar{A}_{0} \bar{N}\right|=2 p^{a}$, some $a \geq 1$. Since this is true for any prime $p$ dividing $\left[B: A_{0}\right]$, this index must be a $p$-power. Hence, by $(4.4), B / C_{B}\left(\left.\left.V_{\lambda}\right|_{A_{0}}\right|^{\otimes B}\right)$ is a $p$-group, concluding Case 1.

Case 2. $\bar{A} \bar{N}$ is noncyclic and $\left.\chi_{\lambda}\right|_{\bar{A}_{0}} ¥ \rho_{\bar{A}_{0}}$.

Since $\left.\chi_{\lambda}\right|_{\bar{A}_{0}} ¥ \rho_{\bar{A}_{0}}$, then $\bar{A}_{0}(\leq \widehat{A}), \bar{N}, R$, and $V_{\lambda}$ are one of the exceptional cases of Theorem (3.15). Notice that $\mathscr{O}_{2}(\widehat{A})$ is nonabelian and $\mathscr{O}_{2^{\prime}}(\widehat{A})$ is cyclic (or trivial). We may view $V_{\lambda}$ as an $\hat{A}$-module. Write $\hat{A}=S \times T \times P$, where $S=\mathscr{O}_{2}(\widehat{A}), T=\mathscr{O}_{\{2, p\}^{\prime}}(\widehat{A})$, and $P=\mathscr{O}_{p}(\widehat{A}) .(T=1$ and $P=1$ are possible. $)$ Recall that in all of the exceptional cases of (3.14) $\left.\chi_{\lambda}\right|_{\widehat{A}} \geq\left(\rho_{\widehat{A}}-\rho_{\widehat{A} \mid \widehat{A}^{\prime}}\right) / 2=$ $\varphi \rho_{T} \rho_{P}$, where $\varphi=\left(\rho_{S}-\rho_{S / S^{\prime}}\right) / 2$. Let $V$ be the $\hat{A}$-module with character $\varphi \rho_{T} \rho_{P}$.

By (4.3), $\bar{C}$ acts on $\left.\left.V\right|_{A_{0}}\right|^{\otimes C}$ as a subgroup of

$$
\mathscr{O}_{p^{\prime}}\left(\bar{A}_{0}\right) \times\left(\mathscr{O}_{p}\left(\bar{A}_{0}\right) \sim Z_{p}\right)=S \times T \times\left(P \sim Z_{p}\right)
$$

acting on $V^{(p)}$. Let $\Phi$ be the character of the $\left(S \times T \times\left(P \sim Z_{p}\right)\right)$-module $V^{(p)}$. Since $V_{\lambda} \geq V, \psi=\left.\chi_{\lambda}\right|_{\bar{A}_{0}}{ }^{\otimes C} \geq 3 \rho_{\bar{C}}$ will follow from $\Phi \geq 3 \rho_{S \times T \times\left(P \sim Z_{p}\right)}$. We will show the latter.

Let $a=|T|, b=|P|, c=\left[S: S^{\prime}\right] / 2$, and $d=\left|S^{\prime}\right|$. Observe (from (3.15)) that $c \geq 2, d \geq 4$, and $|S|=2 c d$. For $x \in S \times T \times P$,

$$
\varphi \rho_{T} \rho_{P}(x)= \begin{cases}a b c(d-1), & x=1, \\ -a b c, & x \in\left(S^{\prime}\right)^{\#}, \\ 0, & \text { elsewhere. }\end{cases}
$$

Set $D=\left(P \sim Z_{p}\right)^{\prime} \cup\left\{x \in\left(P \sim Z_{p}\right) \backslash P^{(p)}:|x|=p\right\}$, a subgroup of $P \sim Z_{p}$. By (4.5), for $x \in S \times T \times\left(P \sim Z_{p}\right)$,

$$
\Phi(x)= \begin{cases}{[a b c(d-1)]^{p},} & x=1, \\ a b c(d-1), & x \in D \backslash\left(P \sim Z_{p}\right)^{\prime}, \\ -a b c & x \in\left(S^{\prime}\right)^{\#}\left(D \backslash\left(P \sim Z_{p}\right)^{\prime}\right), \\ -(a b c)^{p}, & x \in\left(S^{\prime}\right)^{\#}, \\ 0, & \text { elsewhere. }\end{cases}
$$

A routine calculation shows that

$$
\begin{aligned}
\Phi= & e \rho_{S \times T \times\left(P \sim Z_{p}\right)}+\left[\frac{1}{2}\left(\rho_{S}-\rho_{S / S^{\prime}}\right)\right] \\
& \times \rho_{T}\left[f \rho_{P \sim Z_{p}}+\rho_{P \sim Z_{p} / D}+\left(\rho_{P \sim Z_{p}}-\rho_{\left.\left.P \sim Z_{p} /\left(P \sim Z_{p}\right)^{\prime}\right)\right],}\right.\right.
\end{aligned}
$$

where $e=(a c)^{p-1}(d-1)\left[(d-1)^{p-1}-1\right] / 2 p d$ and $f=\left[(a c)^{p-1}-1\right] / p$ are integers. Recalling that $c \geq 2, d \geq 4$, and $p \geq 3$, we have

$$
e \geq(a c)^{p-1}(d-1) / 2 \geq 2^{2} 3 / 2 \geq 3 \text {. }
$$

Thus, $\Phi \geq 3 \rho_{S \times T \times\left(P \sim Z_{p}\right)}$ and so $\psi \geq 3 \rho_{C}^{*}$, a contradiction, concluding the proof.

We modify the argument slightly to obtain 
(6.3) Corollary. Assume Hypothesis (6.1). Let $n>1$ with $(n,|A R|)=1$. Set $V=V_{\lambda}^{(n)}$, an $\left(A \sim Z_{n}\right)$-module. We can view $V$ as a module for $A \simeq A^{\Delta} \leq$ $A \sim Z_{n}$. Then $V$ contains three copies of the regular ${ }^{*}$ A-module.

Proof. Clearly it suffices to consider the case when $n=p$, a prime. Set $B=$ $A \times C$ with $C \simeq Z_{p}$. Now $B$ acts on $\left.\left.V_{\lambda}\right|_{A}\right|^{\otimes B}$ like $A^{\Delta} \times Z_{p} \leq A \sim Z_{p}$ acting on $V_{\lambda}^{(p)}$. First assume that $p$ is odd. Then the hypotheses of Theorem (6.2) are satisfied with the exception that here we may not have $(|B|,|N|)=1$. This hypothesis was used only in Case 1 to obtain $p \geq 5$ when $|\bar{N}|=3$. However, in this situation $V_{\lambda}$ contained two copies of the regular ${ }^{*} A$-module and we had $r|A|=r\left(r^{m}+1\right) / 3$ which is even, so we still have $p$ odd and hence $p \geq 3$. Then, by (5.3), $V_{\lambda}^{(p)}$ contains two copies of the regular* $\left(B=A^{\Delta} \times Z_{p}\right)$-module and so contains $2 p \geq 3$ copies of the regular* $\left(A \simeq A^{\Delta}\right)$-module.

We next assume $p=2$. Then $|A R|$ is odd and, by Theorem (3.15), $V_{\lambda}$ contains the regular ${ }^{*} A$-module and hence $V_{\lambda}^{(2)}$ contains at least $\operatorname{dim} V_{\lambda}=$ $r^{m} \geq 3$ copies of the regular* $A$-module.

(6.4) Theorem. Suppose $G=A N R$ satisfies Hypothesis (6.1) and that in addition $\bar{R}=R / Z(R)$ is faithful. Assurne $A_{0}<A$. Then $\left.\left.\chi_{\lambda}\right|_{A_{0}}\right|^{\otimes A} \geq \rho_{A}$.

Proof. Write $V_{\lambda}=V_{\lambda}(A N R)$ and $\chi_{\lambda}=\chi_{\lambda}(A N R)$. Suppose $\chi_{\lambda} \geq \rho_{A_{0}}$. If $|A|$ is not a prime power, the result follows from (5.8), while if it is, the result follows from (5.7) and induction on $\left[A: A_{0}\right]$. Then we may assume that $\left.\chi_{\lambda}\right|_{A_{0}} \ngtr \rho_{A_{0}}$ and hence that $A_{0}<A \leq \widehat{A}, N$, and $R$ are one of the exceptional cases of Theorem (3.15). Also, by Theorem (6.2), if $\left[A: A_{0}\right]$ is divisible by an odd prime, then $\left.\left.\chi_{\lambda}\right|_{A_{0}}\right|^{\otimes A} \geq 3 \rho_{A}$ and so we may assume that $\left[A: A_{0}\right]$ is a power of 2. Further, by the argument above, if the result holds for $\left[A: A_{0}\right]=2$ it holds in general. Thus, it suffices to consider only those exceptions of (3.15) which have $\left[A: A_{0}\right]=2$. (But $\left[\widehat{A}: A_{0}\right]$ may be greater than 2.) The values of $\chi_{\lambda}$ are given there explicitly and the decomposition into irreducible characters is partially described. We divide the exceptions into two groups.

Case 1. $r^{m}=81, A=\widehat{A}=\langle h, k, i\rangle$ is the group of (2), and $A_{0}$ is either $\left\langle h^{2}, h k, i\right\rangle$ or $\left\langle h^{2}, h k, k i\right\rangle$. (Notice that these are the only possibilities for $A_{0}$ which contain elements $a$ for which $\chi_{\lambda}(a)=-3$.)

Since $\left.\chi_{\lambda}\right|_{\widehat{A}} \geq\left(\rho_{\widehat{A}}-\rho_{\widehat{A} / \widehat{A}^{\prime}}\right) / 2$, it follows from Frobenius reciprocity that $\left.\chi_{\lambda}\right|_{A_{0}} \geq \varphi=\left(\rho_{A_{0}}-\rho_{A_{0} / A_{0}^{\prime}}\right)$. Then it suffices to show that $\left.\varphi\right|^{\otimes A} \geq \rho_{A}$. Set $c=\left[A_{0}: A_{0}^{\prime}\right]$. Then for $a \in A_{0}$,

$$
\varphi(a)= \begin{cases}64-c, & a=1, \\ -c, & a \in\left(A_{0}^{\prime}\right)^{\#}, \\ 0, & a \in A_{0} \backslash A_{0}^{\prime} .\end{cases}
$$

By (4.6), $\left.\varphi\right|^{\otimes A}(a)=\varphi(a)^{2}$ for $a \in A_{0}$, while $\left.\varphi\right|^{\otimes A}(a)=\varphi\left(a^{2}\right)$ for $a \in A \backslash A_{0}$. Noticing that $A \backslash A_{0}$ contains no involutions, we see that for $a \in A$,

$$
\left.\varphi\right|^{\otimes A}(a)= \begin{cases}(64-c)^{2}, & a=1, \\ c^{2}, & a \in\left(A_{0}^{\prime}\right)^{\#}, \\ -c, & a \in H \backslash G, \\ 0, & \text { elsewhere, }\end{cases}
$$


and $\left.\varphi\right|^{\otimes A}=d \rho_{A}+(e+4) \rho_{A / A_{0}^{\prime}}-2 \rho_{A / G}-2 \rho_{A / H}$, where we have one of the following cases:

\begin{tabular}{c|ccccc}
$A_{0}$ & $A_{0}^{\prime}$ & $H$ & $G$ & $c$ & $d e$ \\
\hline$\left\langle h^{2}, h k, i\right\rangle$ & $\left\langle h^{2}\right\rangle$ & $\left\langle h, k, i^{2}\right\rangle$ & $\left\langle h^{2}, h k, i^{2}\right\rangle$ & 8 & 242 \\
$\left\langle h^{2}, h k, h i\right\rangle$ & $\left\langle h^{4}\right\rangle$ & $\left\langle h^{2}, k, i^{2}\right\rangle$ & $\left\langle h^{2}, i^{2}\right\rangle$ & 16 & 16
\end{tabular}

Thus, $\left.\left.\chi_{\lambda}\right|_{A_{0}}\right|^{\otimes A} \geq\left.\varphi\right|^{\otimes A} \geq \rho_{A}$, as desired.

Case 2. All other cases.

In the remaining cases, $m=2 n, r^{n} \geq 5$, and for $a \in A_{0}$,

$$
\chi_{\lambda}(a)= \begin{cases}r^{2 n}, & a=1, \\ \pm r^{n}, & a \in A_{0} \backslash Z(A), a^{2}=1, \\ 1, & a \in\left(A_{0}^{2}\right)^{\#}, \\ \pm 1, & \text { elsewhere. }\end{cases}
$$

Then, by (4.6), for $a \in A$,

$$
\left.\left.\chi_{\lambda}\right|_{A_{0}}\right|^{\otimes A}(a)= \begin{cases}r^{4 n}, & a=1, \\ r^{2 n}, & a \in A \backslash Z(A), a^{2}=1, \\ 1, & \text { elsewhere. }\end{cases}
$$

In all cases we can write

$$
\left\{a \in A \backslash Z(A): a^{2}=1\right\}=\left(E \backslash \widehat{A^{\prime}}\right) \cup(F \backslash G),
$$

where $E=\left\langle h^{2}, h k\right\rangle, \widehat{A^{\prime}}=\left\langle h^{2}\right\rangle, G=\langle-1\rangle$, and $F$ is one of $\left\langle i^{2},-1\right\rangle$, $\langle j,-1\rangle$, or $\langle-1\rangle$ (e.g. if $\left.i^{2} \in A, F=\left\langle i^{2},-1\right\rangle\right)$. Also, in all cases $|A|$ divides $8\left(r^{2 n}-1\right)$ and $\left|\widehat{A}^{\prime}\right|=r^{n} \pm 1$. Let $\mu$ be an irreducible $A$-character. Routine calculations then show that

$$
\begin{aligned}
|A|\left(\left.\left.\chi_{\lambda}\right|_{A_{0}}\right|^{\otimes A}, \mu\right)= & \left(r^{4 n}-1\right) \mu(1)-\left(r^{2 n}-1\right) \mu(1) \\
& \times\left[|E| \delta_{\left.1 \mu\right|_{E}}-\left|A^{\prime}\right| \delta_{\left.1 \mu\right|_{A^{\prime}}}+|F| \delta_{\left.1 \mu\right|_{F}}-|G| \delta_{\left.1 \mu\right|_{G}}\right]+|A| \delta_{1 \mu} \\
\geq & \left(r^{4 n}-1\right) \mu(1)-\left(r^{2 n}-1\right) \mu(1)\left[\left(r^{n}+1\right)+2\right] \\
= & \mu(1)\left(r^{2 n}-1\right)\left(r^{n}-2\right)\left(r^{n}+1\right),
\end{aligned}
$$

and so $\left.\left.\chi_{\lambda}\right|_{A_{0}}\right|^{\otimes A} \geq \mu(1)\left(r^{n}-2\right)\left(r^{n}+1\right) / 8 \geq \mu(1)$. Thus, $\left.\left.\chi_{\lambda}\right|_{A_{0}}\right|^{\otimes A} \geq \rho_{A}$, as desired.

The crucial facts used in the above proof were the descriptions of the character $\left.\chi_{\lambda}\right|_{A}$ when $\left.\chi_{\lambda}\right|_{A} \nsucceq \rho_{A}$ and of the noncentral involutions in this situation. Using these facts and modifying the proof slightly we obtain the following.

(6.5) Theorem. Assume Hypothesis (6.1) and that $|\bar{N}| \neq 2$ and $\bar{R}$ is faithful. Let $A_{0} \leq A$ and write $\chi_{\lambda}=\chi_{\lambda}(A N R)$. Set $\chi=\chi_{\lambda}^{(2)}$, an $A_{0}^{\Delta} \times Z_{2} \leq A_{0} \sim Z_{2}$ character. Then $\chi \geq 3 \rho_{A_{0}^{\Delta} \times Z_{2}}$.

Proof. If $\left.\chi_{\lambda}\right|_{A_{0}} \geq 3 \rho_{A_{0}}$, the result follows from (5.5). Then we may assume that $A_{0}, A, N, R$, and $V_{\lambda}$ are one of the exceptional cases of (3.13). Assume $\left.\chi_{\lambda}\right|_{A_{0}} \geq \rho_{A_{0}}$. If $\left|A_{0}\right|$ is not a power of 2 , the result follows from (5.8), while if it 
is a 2 power, it follows from (5.7) $\left(p=2\right.$ and either $|\bar{H}|=\left|A_{0}\right|>2$ or $\left.\chi_{\lambda}\right|_{A_{0}} \geq$ $\left.2 \rho_{A_{0}}\right)$. Then we may assume that $\left.\chi_{\lambda}\right|_{A_{0}} \nsucceq \rho_{A_{0}}$ and hence that we have one of the exceptions of (3.15). Clearly we may assume $A_{0}=A=\widehat{A}$. For convenience we identify $A^{\Delta}$ with $A$ and write $Z_{2}=\langle y\rangle$ so that $A^{\Delta} \times Z_{2}=A \cup A y$.

Case 1. $A=\langle h, k, i\rangle$ is the group of (2).

Now $\left.\chi_{\lambda}\right|_{A} \geq \varphi=\left(\rho_{A}-\rho_{A / A^{\prime}}\right) / 2$ and for $a \in A$,

$$
\varphi(a)= \begin{cases}56, & a=1, \\ -8, & a \in\left(A^{\prime}\right)^{\#}, \\ 0, & a \in A \backslash A^{\prime} .\end{cases}
$$

The involutions of $A y$ are $\left[\left(E \backslash A^{\prime}\right) \cup F\right] y$, where $E=\left\langle h^{2}, h k\right\rangle$ and $F=$ $\left\langle i^{2},-1\right\rangle$. Also, $\left\{x \in A y: x^{2} \in A^{\prime}\right\}=G y$, where $G=\left\langle h, k, i^{2}\right\rangle$. Then for $x \in A \times Z_{2}$,

$$
\left.\varphi\right|^{\otimes A Z_{2}}(x)= \begin{cases}3136, & x=1, \\ 64, & x \in\left(A^{\prime}\right)^{\#}, \\ 56, & x \in\left[\left(E \backslash A^{\prime}\right) \cup F\right] y, \\ -8, & x \in\left(G \backslash\left[\left(E \backslash A^{\prime}\right) \cup F\right]\right) y, \\ 0, & \text { elsewhere. }\end{cases}
$$

Let $\nu$ be the nontrivial $Z_{2}$-character. Routine calculations show that

$$
\left.\varphi\right|^{\otimes A Z_{2}}=12 \rho_{A Z_{2}}+\left(1_{Z_{2}}-\nu\right)\left(\rho_{A / F}+4 \rho_{A / E}-2 \rho_{A / G}\right)+4 \nu \rho_{A / A^{\prime}} \geq 3 \rho_{A Z_{2}} .
$$

Case 2. All other cases.

As before

$$
\left.\left.\chi_{\lambda}\right|_{A}\right|^{\otimes A Z_{2}}(a)= \begin{cases}r^{4 n}, & a=1, \\ r^{2 n}, & a \in A \backslash Z(A), a^{2}=1, \\ 1, & \text { elsewhere, }\end{cases}
$$

and we can write $\left\{x \in A Z_{2} \backslash Z(A): x^{2}=1\right\}=\left(E \backslash A^{\prime}\right) \cup(F \backslash G)$, where now $E=\left\langle h^{2}, h k\right\rangle Z_{2}, A^{\prime}=\left\langle h^{2}\right\rangle, G=\langle-1\rangle$, and $F$ is either $\langle-1\rangle Z_{2}$ or $\langle-1, j\rangle Z_{2}$. For $\mu$ an irreducible $A Z_{2}$-character,

$$
\left(\left.\left.\chi_{\lambda}\right|_{A}\right|^{\otimes A Z_{2}}, \mu\right) \geq|N| \mu(1)\left(r^{n}-2\right)\left(r^{n}+1\right) / 8 \geq 3 \rho_{A}
$$

and $\left.\left.\chi_{\lambda}\right|_{A}\right|^{\otimes A Z_{2}} \geq 3 \rho_{A}$, as desired.

In Theorem (6.4) we looked at the module $\left.\left.V_{\lambda}(A N R)\right|_{A_{0}}\right|^{\otimes A}, A_{0}<A$, when $\bar{R}$ was a faithful $A N$-module. We now want to consider the general case.

(6.6) Theorem. Assume Hypothesis (6.1). Let $A_{0}<A$. Then $\left.\left.\chi_{\lambda}(A N R)\right|_{A_{0}}\right|^{\otimes A} \geq$ $\rho_{A}^{*}$.

Proof. Write $V_{\lambda}=V_{\lambda}(A N R)$ and $\chi_{\lambda}=\chi_{\lambda}(A N R)$. Set $\bar{A}=A / C_{A}\left(V_{\lambda}\right), \bar{A}_{0}=$ $A_{0} / C_{A_{0}}\left(V_{\lambda}\right) \simeq A_{0} C_{A}\left(V_{\lambda}\right) / C_{A}\left(V_{\lambda}\right)$, and $\bar{N}=N / C_{N}\left(V_{\lambda}\right)$. If $\left.\chi_{\lambda}\right|_{\bar{A}_{0}} \geq 3 \rho_{\bar{A}_{0}}$, the result follows from (5.5) and so we may assume that $\bar{A}_{0}, \bar{A}, \bar{N}$, and $R$ are one of the exceptional cases of (3.13). Also, if $C_{A}\left(V_{\lambda}\right) \leq A_{0}$, then the result follows from (6.4). Then we may assume $A_{0}<A_{0} C_{A}\left(V_{\lambda}\right)$.

Choose $A_{0} \triangleleft B \leq A_{0} C_{A}\left(V_{\lambda}\right)$ with [ $\left.B: A_{0}\right]=p$, a prime. By (5.5), it suffices to show that $\left.\left.\chi_{\lambda}\right|_{A_{0}}\right|^{\otimes B} \geq 3 \rho_{B}^{*}$. This follows from (6.2) except when either (i) 
$p=2$ or (ii) $p$ is odd, $\left|\bar{A}_{0}\right|=p^{a}$ for some positive integer $a$, and $\left.\chi_{\lambda}\right|_{A_{0}} \geq \rho_{A_{0}}^{*}$. Now by (4.2), $B / C_{B}\left(\left.\left.V_{\lambda}\right|_{A_{0}}\right|^{\otimes B}\right)$ acts on $\left.\left.V_{\lambda}\right|_{A_{0}}\right|^{\otimes B}$ like $\bar{A}_{0}^{\Delta} \times Z_{p}$ acting on $V_{\lambda} \mid \frac{(p)}{A_{0}}$. The result now follows from (6.5) when $p=2$ and from (5.7) when $p$ is odd.

(6.7) Theorem. Let $R \triangleleft A M R$, where $R$ is an extraspecial group, $M \triangleleft A M, A$ is nilpotent, and

$$
(|A M|,|R|)=(|A|,|M|)=1 .
$$

Assume $Z(R) \leq Z(A M R)$ and that $\bar{R}=R / Z(R)$ is a faithful, irreducible AM-module. By (3.6), $\left.\bar{R} \simeq \bar{R}_{0}\right|^{A M}$ for some form quasiprimitive $A_{0} N$-module, where $A_{0} \leq A$ and $N \leq M$. Suppose $N<M$ and $1 \neq N / C_{N}\left(\bar{R}_{0}\right)$ is cyclic. Then $\left.\chi_{\lambda}(A M R)\right|_{A} \geq 3 \rho_{A}$.

Proof. Write $V_{\lambda}=\left.V_{\lambda}\left(A_{0} N R_{0}\right)\right|_{A_{0} N}$ and $\chi_{\lambda}=\left.\chi_{\lambda}\left(A_{0} N R_{0}\right)\right|_{A_{0} N}$. By (5.1),

$$
\left.\left.V_{\lambda}(A M R)\right|_{A M} \simeq W \otimes V_{\lambda}\right|^{\otimes A M},
$$

where $\operatorname{dim} W=1$. When counting regular modules we may assume that $W$ is the trivial module so that $\left.\left.V_{\lambda}(A M R)\right|_{A M} \simeq V_{\lambda}\right|^{\otimes A M}$. Further, since $\left.\left.V_{\lambda}\right|^{\otimes A M}\right|_{A}=$ $\left.V_{\lambda}\right|^{\otimes A_{0} M}\left|A_{A_{0}}\right|^{\otimes A}$, by (5.5) it suffices to show that $\left.\left.V_{\lambda}\right|^{\otimes A_{0} M}\right|_{A_{0}}$ contains three copies of the regular ${ }^{*}$ module. That is, if the theorem is true for $A=A_{0}$, then it is true in general. In order to reduce the number of subscripts we then assume that $A_{0}=A$.

Write $V=\left.V_{\lambda}(A M R)\right|_{A M}$ and $\chi=\left.\chi_{\lambda}(A M R)\right|_{A M}$. By Lemma (5.12), $V \simeq$ $\left.\left.\Pi^{\otimes} V_{\lambda}\right|_{C_{A}\left(x_{i}\right)}\right|^{\otimes A}$, where the $x_{i}$ 's are appropriately chosen double $(A, A N)$-coset representatives in $A M$. Set $S=\left\{i: C_{A}\left(x_{i}\right)<A\right\}, T=\left\{i: C_{A}\left(x_{i}\right)=A\right\}, V_{S}=$ $\left.\left.\prod_{i \in S}^{\otimes} V_{\lambda}\right|_{C_{A}\left(x_{i}\right)}\right|^{\otimes A}$ and $V_{T}=\left.\left.\prod_{i \in T}^{\otimes} V_{\lambda}\right|_{C_{A}\left(x_{i}\right)}\right|^{\otimes A}=\left.V_{\lambda}\right|_{A} ^{(|T|)}$. Then $V=V_{S} \otimes V_{T}$. Also, we may take $x_{1}=1$ and so $T \neq \varnothing$.

We first assume $S=\varnothing$. Then the number of double coset representatives is $|T|=[M: N]$ and by $(6.3) \chi=\chi_{\lambda}^{(|T|)} \geq 3 \rho_{A}$, as desired. We next assume $S \neq \varnothing$. By (6.6), $\left.\left.V_{\lambda}\right|_{C_{A}\left(x_{i}\right)}\right|^{\otimes A}$ contains a copy of the regular ${ }^{*} A$-module, all $i \in S$, and so by (5.9), $V_{S}=\prod_{i \in S}^{\otimes} V_{\lambda}\left|C_{A}\left(x_{i}\right)\right|^{\otimes A}$ contains a regular $A / C_{A}\left(V_{S}\right)$ module. But $C_{A}\left(V_{S}\right) \leq C_{A}\left(\left.V_{\lambda}\right|_{A}\right) \leq C_{A}\left(V_{T}\right)$ and so $C_{A}\left(V_{S}\right)=C_{A}(V)=(0)$. Thus, $V_{S}$ contains the regular $A$-module and then $V=V_{S} \otimes V_{T}$ contains $\operatorname{dim} V_{T}=\left(\operatorname{dim} V_{\lambda}\right)|T|$ copies of the regular $A$-module. Then we may assume $\left(\operatorname{dim} V_{\lambda}\right)|T|<3$, i.e., $\operatorname{dim} V_{\lambda}=2$ and $|T|=1$. But $\operatorname{dim} V_{\lambda}=r^{m}$, where $\left|R_{0}\right|=r^{2 m+1}$. Hence $\left|R_{0}\right|=8$ and $\bar{R}_{0} \simeq Z_{2} \times Z_{2}$. Now $A N / C_{A N}\left(\bar{R}_{0}\right)$ embeds into $\operatorname{Aut}\left(\bar{R}_{0}\right) \simeq Z_{3}$ and $N / C_{N}\left(\bar{R}_{0}\right) \neq 1$. Therefore $A=C_{A}\left(\bar{R}_{0}\right)=C_{A}\left(V_{\lambda}\right)$ and $A$ is trivial on $V_{\lambda}$. Then for all $i \in S \cup T,\left.\chi_{\lambda}\right|_{C_{A}\left(x_{i}\right)}=2 \rho_{C_{A}\left(x_{i}\right)}^{*}$. Since $|A|$ is odd $(r=2)$, by (5.5), $\chi_{\lambda}\left|C_{A}\left(x_{i}\right)\right|^{\otimes A} \geq 2 \rho_{A}^{*}$ and by (5.9), $\chi=\left.\left.\prod_{i}^{\otimes} \chi_{\lambda}\right|_{C_{A}\left(x_{i}\right)}\right|^{\otimes A} \geq$ $2^{|S \cup T|} \rho_{A} \geq 3 \rho_{A}$, as desired.

We are now ready to prove the main theorem. We phrase the hypotheses in a more general form than in the introduction. (In fact, they turn out to be equivalent.)

(6.8) Theorem. Suppose $G=A M R$, where $R \triangleleft A M R$ is an extraspecial $r$ group, $r$ is a prime, $1 \neq M \triangleleft A M$ is solvable, $A$ is nilpotent, and $(|A|,|M| r)=$ $(|M|, r)=1$. Suppose $Z(R) \leq Z(A M R)$ and $\bar{R}=R / Z(R)$ is a faithful, irreducible AM-module. By (3.6), $\left.\bar{R} \simeq \bar{R}_{0}\right|^{A M}$, where $\bar{R}_{0}$ is a form primitive $A_{0} N$-module, $A_{0} \leq A$, and $N \leq M$. Suppose $N / C_{N}\left(\bar{R}_{0}\right)$ is abelian. Let $\lambda \neq 1$ 
be an irreducible $Z(R)$-character. Let $V_{\lambda}=V_{\lambda}(A M R)$ and $\chi_{\lambda}=\chi_{\lambda}(A M R)$ be the module and character of (2.2). Then $\chi_{\lambda} \geq 3 \rho_{A}$ unless $M$ is abelian and one of the following occurs:

(1) $M$ is cyclic, $\bar{R}$ is a form primitive $A M$-module, and $A, M$, and $R$ are one of the exceptional cases of Theorem (3.13),

(2) $A$ is a p-group, $p$ an odd prime, $r^{a}=2 p^{b}-1$, for some positive integers $a, b$, and $\exp (M)=2$,

(3) $r=2^{a}+1$, some $a, \mathscr{O}_{2^{\prime}}(A)$ is cyclic, and both $\left|\mathscr{O}_{2^{\prime}}(A)\right|$ and $\exp (M)$ divide $(r+1) / 2$, or

(4) $A$ is a 2-group and one of the following occurs:

(i) $\exp (M)=3$ and $r=3 \cdot 2^{a} \pm 1$, some $a$,

(ii) $\exp (M)=5$ and either $r=3$ or $r=5 \cdot 2^{a} \pm 1$, some $a$,

(iii) $\exp (M)=15$ and $r=11$, or

(iv) $r=2^{a}-1$ and $\exp (M)$ divides $(r-1) / 2$.

Proof. Since $\bar{R}_{0}$ is form quasiprimitive and $N \triangleleft A_{0} N$, either $\left.\bar{R}_{0}\right|_{N}$ is homogeneous or $\left.\bar{R}_{0}\right|_{N}=\bar{R}_{1} \dot{+} \bar{R}_{2}$, the sum of two homogeneous components on which $N$ acts contragrediently. In the latter case $C_{N}\left(\bar{R}_{1}\right)=C_{N}\left(\bar{R}_{2}\right)=C_{N}\left(\bar{R}_{0}\right)$. Thus, since $N / C_{N}\left(\bar{R}_{0}\right)$ is abelian, in both cases it is in fact cyclic. Suppose $N=C_{N}\left(\bar{R}_{0}\right)$. Then $W=C_{\left.\bar{R}_{0}\right|^{A_{0} M}}(M) \neq(0)$ is an $A_{0} M$-submodule of $\left.\bar{R}_{0}\right|^{A_{0} M}$ and $(0) \neq\left. W\right|^{A M}$ is a submodule of $\bar{R}=\left.\bar{R}_{0}\right|^{A M}$. The latter is irreducible and so $\bar{R}=\left.W\right|^{A M}$. However, since $M \triangleleft A M, M$ centralizes $\left.W\right|^{A M}$, contradicting the assumption that $\bar{R}$ is a faithful $A M$-module. Then $N \neq C_{N}\left(\bar{R}_{0}\right)$ and hence $A_{0}$ (= " $\left.A "\right), N$, and $R_{0}$ (= " $R$ ") satisfy Hypothesis (6.1).

We assume we have a counterexample to the theorem, in particular, that $\chi_{\lambda} \geq$ $3 \rho_{A}$. Then by (6.7), $N=M$. Set $\bar{M}=M / C_{M}\left(\bar{R}_{0}\right)$ and $\bar{A}_{0}=A_{0} / C_{A_{0}}\left(\bar{R}_{0}\right)$. Since $C_{A_{0}}\left(\bar{R}_{0}\right) \leq C_{A_{0}}(M), \bar{A}_{0}$ acts on $\bar{M}$ and $A_{0} M / C_{A_{0} M}\left(\bar{R}_{0}\right) \simeq \bar{A}_{0} \bar{M}$. Let $\bar{\varphi}: A M \rightarrow \bar{A}_{0} \bar{M} \sim \widehat{A}$ be the monomorphism of $\S 4$ (which does not depend on the module $U)$. Now $\bar{\varphi}(M)$ is a Hall $\pi(M)$-subgroup of $\bar{A}_{0} \bar{M} \sim \hat{A}$ and $\bar{M}^{\left(\left[A: A_{0}\right]\right)}$ is one such subgroup. Then

Step 1. $M=N$ is abelian, $1 \neq \bar{M}=M / C_{M}\left(\bar{R}_{0}\right)$ is cyclic, and $\exp (M)=$ $\exp (\bar{M})$.

If $A=A_{0}$, then $\bar{R}$ is quasiprimitive and we have exception (1). Thus, $A_{0}<A$. If $\left[A: A_{0}\right]$ is divisible by an odd prime, by (6.2), we have exception (2). Thus,

Step 2. $1 \neq\left[A: A_{0}\right]$ is a power of 2 .

By (5.1), $\left.\chi_{\lambda}(A M R)\right|_{A}=\left.\left.\mu \cdot \chi_{\lambda}\left(A_{0} M R_{0}\right)\right|_{A_{0}}\right|^{\otimes A}$ for some linear character $\mu$. When counting regular characters we may assume $\mu=1$. Then, by (5.5), $\left.\chi_{\lambda}\left(A_{0} M R_{0}\right)\right|_{A_{0}} ¥ 3 \rho_{A_{0}}^{*}$ and hence

Step 3. $\bar{A}_{0}, \bar{M}$, and $R_{0}$ are one of the exceptions of (3.13).

Suppose $\left|\bar{A}_{0}\right|$ is not a 2-power. Inspecting the entries of Table (3.14) we see that one of the following happens:

(i) $\left.\chi_{\lambda}\left(A_{0} M R_{0}\right)\right|_{A_{0}} \geq \rho_{\bar{A}_{0}}$ and $\mathscr{O}_{2^{\prime}}\left(\bar{A}_{0}\right)>5$,

(ii) $\left.\chi_{\lambda}\left(A_{0} M R_{0}\right)\right|_{A_{0}} \geq 2 \rho_{\bar{A}_{0}}$, or

(iii) we have entry (4) of Table (3.14). 
(Entry (1) cannot occur since 2||$A \mid$ and $(|A|,|N|)=1$.) In the first two cases, by (5.8) and (5.5), $\left.\chi_{\lambda}\right|_{A}=\left.\left.\chi_{\lambda}\left(A_{0} M R_{0}\right)\right|_{A_{0}}\right|^{\otimes A} \geq 3 \rho_{A}$, a contradiction. Then we must have entry 4$)$ of $(3.14)$. By (4.4), $\mathscr{O}_{2^{\prime}}(A)=\mathscr{O}_{2^{\prime}}\left(\bar{A}_{0}\right)$ and we have exception (3). Finally, suppose $\bar{A}_{0}$ is a 2-group. By (4.4), $A$ is also a 2-group and it follows immediately that we have one of exceptions (3) or (4).

\section{BIBLIOGRAPHY}

1. T. R. Berger, Hall-Higman type theorems. I, Canad. J. Math. 26 (1974), 513-531.

2. __ Hall-Higman type theorems. II, Trans. Amer. Math. Soc. 205 (1975), 47-69.

3. __ Hall-Higman type theorems. III, Trans. Amer. Math. Soc. 228 (1977), 47-83.

4. __ Hall-Higman type theorems. IV, Proc. Amer. Math. Soc. 37 (1973), 317-325.

5. __, Hall-Higman type theorems. V, Pacific J. Math. 73 (1977), 1-62.

6. __, Hall-Higman type theorems. VI, J. Algebra 51 (1978), 416-424.

7. __ Hall-Higman type theorems. VII, Proc. London Math. Soc. (3) 31 (1975), 21-54.

8. __ Representation theory and solvable groups: Length type problems, Proc. Sympos. Pure Math., Vol. 37, Amer. Math. Soc., Providence, R.I., 1980, pp. 431-441.

9. T. R. Berger, B. B. Hargraves, and C. Shelton, The regular module problem. II, Comm. Algebra 18 (1990), 2611-2631.

10. __ The regular module problem. III, J. Algebra 131 (1990), 74-91.

11. R. W. Carter and P. Fong, The Sylow 2-subgroups of the finite classical groups, J. Algebra 1 (1964), 139-151.

12. D. Gluck and I. M. Isaacs, Tensor induction of generalized characters and permutation groups, Illinois J. Math. 27 (1983), 514-518.

13. D. Gorenstein, Finite groups, Harper \& Row, New York, 1968.

14. B. B. Hargraves, The existence of regular orbits for nilpotent groups, J. Algebra 72 (1981), 54-100.

15. B. Huppert, Endliche Gruppen. I, Springer, Berlin, 1967.

16. I. M. Isaacs, Character theory of finite groups, Academic Press, New York, 1976.

17. A. E. Parks, Generalized permutation characters of solvable groups, J. Algebra 93 (1985), 445-474.

18. D. S. Passman, Groups with normal solvable Hall $p^{\prime}$-subgroups, Trans. Amer. Math. Soc. 123 (1966), 99-111.

19. E. E. Schult, On groups admitting fixed point free abelian operator groups, Illinois J. Math. 9 (1965), 701-720.

20. A. Turell, Supersolvable automorphism groups of solvable groups, Math. Z. 183 (1983), 4773.

21. D. L. Winter, The automorphism group of an extraspecial p-group, Rocky Mountain J. Math. 2 (1972), 159-168.

Department of Mathematics, University of Minnesota, Minneapolis, Minnesota 55455 E-mail address: tom@s5.math.umn.edu

Department of Mathematics, St. Mary's College of Maryland, St. Mary's City, MaryLAND 20686

E-mail address: hargraves_b@vaxa.smcm.edu

Honeywell, Minneapolis, Minnesota 55455 